\title{
Vector Gaussian Multiple Description with Two Levels of Receivers $^{1}$
}

Hua Wang and Pramod Viswanath ${ }^{2}$

\begin{abstract}
The problem of $L$ multiple descriptions of a stationary and ergodic Gaussian source with two levels of receivers is investigated. Each of the first level receivers receive (an arbitrary subset) $k$ of the $L$ descriptions, $(k<L)$. The second level receiver receives all $L$ descriptions. All the receivers, both at the first level and the second level, reconstruct the source using the subset of descriptions they receive. The corresponding reconstructions are subject to quadratic distortion constraints. Our main result is the derivation of an outer bound on the sum rate of the descriptions so that the distortion constraints are met. We show that a natural analog-digital separation architecture involving joint Gaussian vector quantizers and a binning scheme meets this outer bound with equality for several scenarios. These scenarios include the case when the distortion constraints are symmetric and the case for general distortion constraints with $k=2$ and $L=3$. We also show the robustness of this architecture: the distortions achieved are no larger when used to describe any non-Gaussian source with the same covariance matrix.
\end{abstract}

\section{Introduction}

Multiple description coding is used in transmission of information under some quality of service requirement through unreliable communication links. In this problem, (cf. Figure 1), an information source is encoded into $L$ packets, which are sent through $L$ separate parallel communication channels. Some packets may get lost during the transmission, but as long as one packet is received, the decoder can reconstruct the information source with some fidelity, and when more packets are received, the decoder is able to generate higher quality approximations of the information source. In the most general case, there are $2^{L}-1$ different combinations of received packets with each combination corresponding to one of $2^{L}-1$ subsets of $\{1, \ldots, L\}$. In [2], El Gamal and Cover derived an achievable rate region for two descriptions $(L=2)$. This region was shown to be tight for the case of "no excess rate" by Ahlswede [3], and was shown not to be tight in general by Zhang and Berger [5]. In [6-8], a symmetric multiple description problem was studied.

The only known complete solution for the entire rate region is for two descriptions of a memoryless Gaussian source with quadratic distortion measures [1]. This result has been recently gen-

\footnotetext{
${ }^{1}$ This research was sponsored in part by NSF CCR-0325924 and a Vodafone US Foundation Fellowship.

${ }^{2}$ The authors are with the Department of Electrical and Computer Engineering and the Coordinated Science Laboratory, University of Illinois at Urbana-Champaign, Urbana IL 61801; e-mail: \{huawang, pramodv\}@uiuc.edu
} 


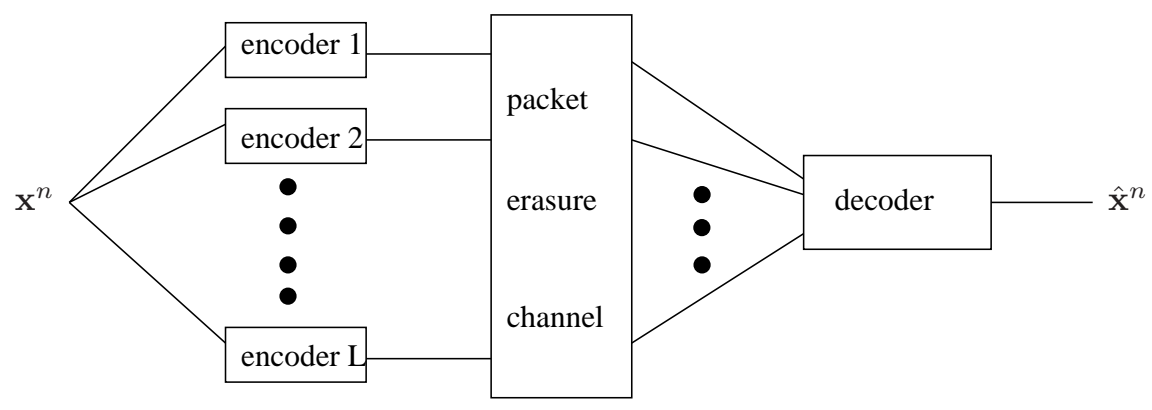

Figure 1: Multiple description problem

eralized to the case of a stationary and ergodic Gaussian source in [11]. Furthermore, the optimal sum rate of $L$ multiple descriptions of a stationary and ergodic Gaussian source with individual and central receivers was also characterized in [11].

In this paper, we generalize the study in [11] by considering the central receiver along with $\left(\begin{array}{l}L \\ k\end{array}\right)$ first level receivers, each of which receives a different subset of $k$ of the $L$ total descriptions $(k<L)$. As in [11], we model the source as a memoryless, but vector Gaussian source. Further, we handle quadratic constraints on the reconstruction of the original stationary and ergodic source by considering a covariance distortion measure constraints, in the sense of a positive semidefinite ordering. Corresponding to $\left(\begin{array}{l}L \\ k\end{array}\right)$ first level receivers and the single central receiver, there are $\left(\begin{array}{l}L \\ k\end{array}\right)+1$ distortion matrices. Specifically, with $k=1$ this problem boils down to the setting studied in detail in [11].

\subsection{A Natural Architecture}

There is a natural achievable architecture which divides the analog and digital aspects of the description problem. As illustrated in Figure 2, the first step is an analog-to-digital conversion and uses $L$ correlated vector quantizers: the source is multiply described by joint Gaussian vector quantizers. In the second step, the digital descriptions are hashed (using the Slepian-Wolf binning scheme) to generate the $L$ indices to be sent through $L$ channels. The hash function (or binning scheme) is in such a way that any subset of the $k$ indices received at first level receiver can uniquely lead to the corresponding $k$ descriptions in the first step. Finally, the reconstruction of the original source is based on these $k$ descriptions. In case all $L$ indices are received (the central receiver), all $L$ descriptions of the first step are available for the source reconstruction. Observe that when $k=1$, there is no second step.

Two important features of this architecture are worth emphasizing: 


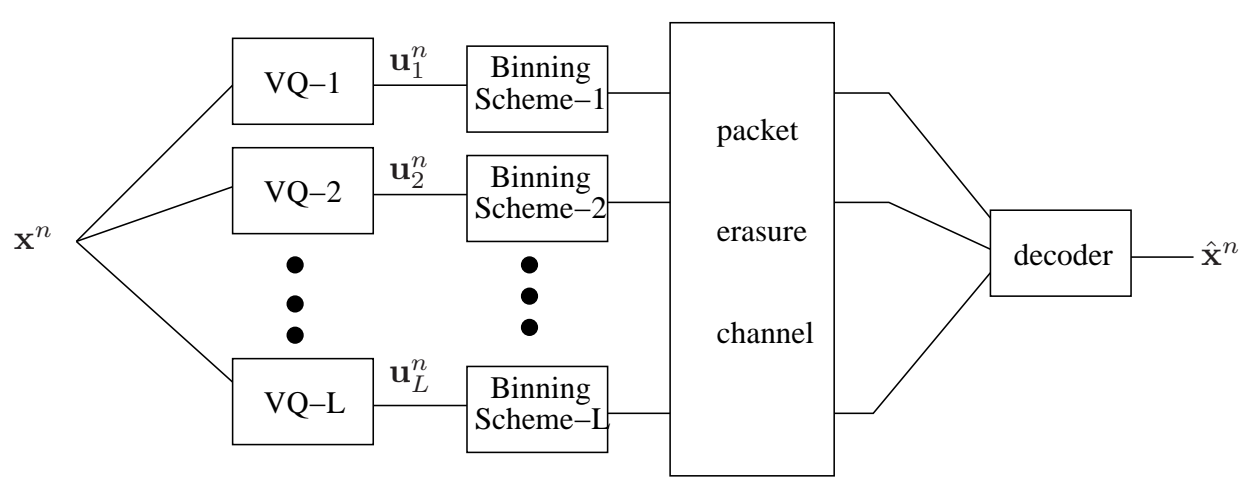

Figure 2: A natural achievable architecture

- It separates the analog and digital aspects of the source representation. The first step converts the original analog source into discrete descriptions (bits). From the view of the second step, any interpretation of the bits in terms of the original real valued source (such as MSB (most significant bit) or LSB (least significant bit)) is irrelevant. Only the statistical relation between the discrete descriptions is used in the binning scheme.

- While the operation in the first step uses the fact that the descriptions are generated at a centralized location, the encoders of the second step could be operating in a distributed fashion without impacting the performance of the architecture; indeed, Slepian-Wolf binning scheme was precisely introduced for distributed lossless compression of discrete information sources. Thus, the flexibility offered by the ability to generate the descriptions at a centralized location appears to be not used in its entirety. Nevertheless, we show the optimality of this architecture in several scenarios in this paper.

We note that this architecture is part of a more general, layered achievable scheme for the general multiple description problem proposed in [8].

\subsection{Main Result}

Our main result is in the derivation of a fundamental lower bound to the sum rate which all descriptions that satisfy the distortion constraint have to obey. We also show that this lower bound is tight in several scenarios by explicitly demonstrating that the natural architecture depicted in Figure 2 achieves this lower bound. These scenarios include the case of symmetric distortion constraints and the case of $k=2$ and $L=3$ for arbitrary distortion constraints. We also show that this natural architecture is robust: for any other memoryless vector source with a fixed covariance matrix, the distortion achieved for the Gaussian source is the largest (in a strong positive semidefinite ordering sense). 
This paper is organized as follows. In Section 2 we give a formal description of the problem. In Section 3 we present a Gaussian achievable scheme. In Section 4 we provide a lower bound to the sum rate In Section 5 we give the conditions for the Gaussian strategy to achieve the lower bound. In Section 6 we study the case when all the distortion constraints for the first level receivers are equal. In Section 7 we study the case when $k=L-1$. In Section 8.1 and 8.2 we study the case where the source is not Gaussian and show the robustness of the natural architecture. In section 8.3 we discuss the problem of multiple description with distributed observations.

We describe the notation in this paper in the following. We use lowercase letters to indicate scalars, boldface lowercase letters to indicate vectors, and boldface uppercase to indicate matrices. The superscript $t$ denotes matrix transpose. We use $\mathbf{I}$ and $\mathbf{0}$ to denote the identity matrix and the all zero matrix respectively. The partial order $\succ(\succcurlyeq)$ denotes positive definite (semidefinite) ordering, i.e., $\mathbf{A} \succ \mathbf{B}(\mathbf{A} \succcurlyeq \mathbf{B})$ means that $\mathbf{A}-\mathbf{B}$ is a positive definite (semidefinite) matrix. We use $\mathcal{N}(\mu, \mathbf{Q})$ to denote a Gaussian random vector with mean $\mu$ and covariance $\mathbf{Q}$. Given $S=\left\{l_{1}, \ldots, l_{k}\right\} \subset\{1, \ldots L\}$, we use $\mathbf{u}_{S}$ to denote $\left(\mathbf{u}_{l_{1}}, \ldots, \mathbf{u}_{l_{k}}\right)$. All logarithms in this paper are to the natural base.

\section{Problem Setting}

We model a stationary and ergodic Gaussian source as a vector (of length $N$ ) memoryless process $\{\mathbf{x}[m]\}_{m}$, with the marginal distribution Gaussian: $\mathcal{N}\left(0, \mathbf{K}_{x}\right)$. Without much loss of generality, we suppose throughout this paper that $\mathbf{K}_{x}$ ( an $N \times N$ matrix) is positive definite. In the multiple description problem of our interest, there are $L$ encoders, each mapping the (analog) information source into a sequence of bits (discrete information). Corresponding to the $l$ th encoder, the encoding function $f_{l}^{(n)}$ maps a source sequence $\mathbf{x}^{n}=\{\mathbf{x}[m]\}_{m=1}^{n}$ to a codeword $f_{l}^{(n)}\left(\mathbf{x}^{n}\right) \in C_{l}^{(n)}$, where $C_{l}^{(n)}$ is the codebook corresponding to this encoder. The rate needed to describe the codebook $C_{l}^{(n)}$ in nats is $R_{l}=\frac{1}{n} \log _{e}\left|C_{l}^{(n)}\right|$.

There are two levels of decoders. For a given $k<L$, there are $\left(\begin{array}{l}L \\ k\end{array}\right)$ first level decoders and one second level central decoder. Each of the first level decoders can receive different combination

of $k$ codewords $f_{l_{1}}^{(n)}\left(\mathbf{x}^{n}\right), \ldots, f_{l_{k}}^{(n)}\left(\mathbf{x}^{n}\right)$. The decoding function then uses the received codewords to reconstruct the original source; we denote the reconstruction by $\hat{\mathbf{x}}_{S}^{n}$, where $S=\left\{l_{1}, \ldots, l_{k}\right\} \subset$ $\{1, \ldots L\}$, parameterizes the specific subset of $k$ codewords (amongst a total of $L$ ) that this particular receiver had access to. The second level central decoder has access to all the $L$ codewords and generates $\hat{\mathbf{x}}_{L}^{n}$, an estimation of the source sequence $\mathbf{x}^{n}$ based on these $L$ codewords. Since we are interested in covariance constraints, the decoding functions can be restricted to be the minimal mean square error (MMSE) estimate of the source sequence based on the received codewords, 
without any loss of generality. Therefore, $\hat{\mathbf{x}}_{S}^{n}$ and $\hat{\mathbf{x}}_{L}^{n}$ can be written as

$$
\begin{aligned}
& \hat{\mathbf{x}}_{S}^{n}=\mathbb{E}\left[\mathbf{x}^{n} \mid f_{l_{1}}^{(n)}\left(\mathbf{x}^{n}\right), \ldots, f_{l_{k}}^{(n)}\left(\mathbf{x}^{n}\right)\right], \quad \forall S=\left\{l_{1}, \ldots, l_{k}\right\} \subset\{1, \ldots L\}, \\
& \hat{\mathbf{x}}_{L}^{n}=\mathbb{E}\left[\mathbf{x}^{n} \mid f_{1}^{(n)}\left(\mathbf{x}^{n}\right), \ldots, f_{L}^{(n)}\left(\mathbf{x}^{n}\right)\right] .
\end{aligned}
$$

We say that the source can be multiple described at rates $\left(R_{1}, \ldots, R_{L}\right)$ with distortion constraints $\mathbf{D}_{S}, S=\left\{l_{1}, \ldots, l_{k}\right\} \subset\{1, \ldots L\}$ and $\mathbf{D}_{L}$, if the following covariance constraints are satisfied

$$
\begin{aligned}
& \frac{1}{n} \sum_{m=1}^{n} \mathbb{E}\left[\left(\mathbf{x}[m]-\hat{\mathbf{x}}_{S}[m]\right)^{t}\left(\mathbf{x}[m]-\hat{\mathbf{x}}_{S}[m]\right)\right] \preccurlyeq \mathbf{D}_{S}, \quad \forall S \subset\{1, \ldots, L\},|S|=k, \\
& \frac{1}{n} \sum_{m=1}^{n} \mathbb{E}\left[\left(\mathbf{x}[m]-\hat{\mathbf{x}}_{L}[m]\right)^{t}\left(\mathbf{x}[m]-\hat{\mathbf{x}}_{L}[m]\right)\right] \preccurlyeq \mathbf{D}_{L} .
\end{aligned}
$$

Note that for the first level decoder there are $\left(\begin{array}{l}L \\ k\end{array}\right)$ distortion constraints.

Our focus is on finding the smallest achievable sum rate of descriptions for given distortion constraints $\mathbf{D}_{S}, \forall S \subset\{1, \ldots, L\},|S|=k$ and $\mathbf{D}_{L}$. For notational simplicity we write these $\left(\begin{array}{l}L \\ k\end{array}\right)+1$ distortion constraints as $\left(\mathbf{D}_{S}, \mathbf{D}_{L}\right)$ in the rest of this paper.

\section{An Achievable Scheme}

We have introduced a natural achievable architecture to address this multiple description problem in Section 1.1 (see Figure 2). In this section, we characterize the performance of this architecture, i.e., the tradeoff between the rate of descriptions and the distortions achieved by the receivers. Let $\mathbf{w}_{1}, \cdots, \mathbf{w}_{L}$ be $N$-dimensional zero-mean jointly Gaussian random vectors that are independent of $\mathbf{x}$. We stack these $L$ vectors together in one vector $\left(\mathbf{w}_{1}, \cdots, \mathbf{w}_{L}\right)$, and use $\mathbf{K}_{w}$ to denote the positive definite covariance matrix of this $L N$-dimensional vector. Define

$$
\mathbf{u}_{l}=\mathbf{x}+\mathbf{w}_{l}, \quad l=1, \ldots, L
$$

We consider those $\mathbf{K}_{w}$ that satisfy the following constraints.

$$
\begin{aligned}
& \operatorname{Cov}\left[\mathbf{x} \mid \mathbf{u}_{S}\right] \stackrel{\text { def }}{=} \mathbb{E}\left[\left(\mathbf{x}-\mathbb{E}\left[\mathbf{x} \mid \mathbf{u}_{S}\right]\right)^{t}\left(\mathbf{x}-\mathbb{E}\left[\mathbf{x} \mid \mathbf{u}_{S}\right]\right)\right] \preccurlyeq \mathbf{D}_{S} \quad \forall S \subset\{1, \ldots, L\},|S|=k, \\
& \operatorname{Cov}\left[\mathbf{x} \mid \mathbf{u}_{1}, \ldots, \mathbf{u}_{L}\right] \stackrel{\text { def }}{=} \mathbb{E}\left[\left(\mathbf{x}-\mathbb{E}\left[\mathbf{x} \mid \mathbf{u}_{1}, \ldots, \mathbf{u}_{L}\right]\right)^{t}\left(\mathbf{x}-\mathbb{E}\left[\mathbf{x} \mid \mathbf{u}_{1}, \ldots, \mathbf{u}_{L}\right]\right)\right] \preccurlyeq \mathbf{D}_{L} .
\end{aligned}
$$

We are now ready to describe the encoding and decoding functions in the architecture of Figure 2 using $\mathbf{K}_{w}$ as a parameter. To construct the codebook for the $l$ th description, first generate $e^{n R_{l}^{\prime}} \mathbf{u}_{l}^{n}$ 
vectors randomly according to the marginal distribution of $\mathbf{u}_{l}$, and then uniformly distribute these $\mathbf{u}_{l}^{n}$ vectors into $e^{n R_{l}}$ bins. As long as

$$
\sum_{l \in T} R_{l}^{\prime} \geq\left[\sum_{l \in T} h\left(\mathbf{u}_{l}\right)\right]-h\left(\mathbf{u}_{T} \mid \mathbf{x}\right), \quad \forall T \subseteq\{1, \ldots, L\}
$$

for every observed source sequence $\mathbf{x}^{n}$, the encoders can find sequences $\left(\mathbf{u}_{1}^{n}, \ldots, \mathbf{u}_{L}^{n}\right)$ that are jointly typical with $\mathrm{x}^{n}$, and send the corresponding bin indices of the resulting $\mathbf{u}_{l}^{n}$ through the $l$ th channel, respectively. Each of the first level receiver receives $k$ descriptions, i.e., $k$ bin indices. It then looks in these $k$ bins for a unique combination of $k$ vectors (each from one bin) that are jointly typical, and generates a reproduction sequence which is the MMSE estimation of the source sequence from these $L$ vectors. The second level receiver receives all $L$ bin indices. It then looks in these $L$ bins for a unique combination of $L$ vectors (each from one bin) that are jointly typical, and generates a reproduction sequence which is the MMSE estimation of the source sequence from these $L$ vectors. Now the probability that a randomly generated combination of codewords $\mathbf{u}_{l}^{n}, l \in S$, for any $S \subseteq\{1, \ldots, L\}$ are jointly typical is roughly

$$
\frac{e^{n h\left(\mathbf{u}_{S}\right)}}{\prod_{l \in S} e^{n h\left(\mathbf{u}_{l}\right)}}
$$

and the number of possible combination of codewords $\mathbf{u}_{l}^{n}, l \in S, S \subseteq\{1, \ldots, L\}$ in a set $S$ of bins are $\prod_{l \in S} e^{n\left(R_{l}^{\prime}-R_{l}\right)}$. Thus, as long as

$$
\sum_{l \in S}\left(R_{l}^{\prime}-R_{l}\right) \leq\left[\sum_{l \in S} h\left(\mathbf{u}_{l}\right)\right]-h\left(\mathbf{u}_{S}\right), \quad \forall S \subset\{1, \ldots L\},|S|=k,
$$

and

$$
\sum_{l=1}^{L}\left(R_{l}^{\prime}-R_{l}\right) \leq\left[\sum_{l=1}^{L} h\left(\mathbf{u}_{l}\right)\right]-h\left(\mathbf{u}_{1}, \ldots, \mathbf{u}_{L}\right),
$$

all the decoders (both the first and the second level) can find unique combination of vectors that are jointly typical.

We now show that the condition in (8) is redundant. To do that, just summing up all the equations in (7) and using the identity

$$
L\left(\begin{array}{l}
L-1 \\
k-1
\end{array}\right)=k\left(\begin{array}{l}
L \\
k
\end{array}\right)
$$

we have

$$
\sum_{l=1}^{L}\left(R_{l}^{\prime}-R_{l}\right) \leq\left[\sum_{l=1}^{L} h\left(\mathbf{u}_{l}\right)\right]-\frac{L}{k\left(\begin{array}{l}
L \\
k
\end{array}\right)} \sum_{S,|S|=k} h\left(\mathbf{u}_{S}\right) \leq\left[\sum_{l=1}^{L} h\left(\mathbf{u}_{l}\right)\right]-h\left(\mathbf{u}_{1}, \ldots, \mathbf{u}_{L}\right),
$$


where in the last step we used the subset inequality of entropy [4, Theorem 16.5.1]. Thus we can see that under condition (7), both level decoders are successful.

The inequalities in Equations (5) and (7) define the region of description rates $\left(R_{1}, \ldots, R_{L}\right)$ that are feasible. In general it appears to be quite involved to exactly evaluate the achievable region by taking union over the intermediary variables $\left(R_{1}^{\prime}, \ldots R_{l}^{\prime}\right)$. In the following, we provide a lower bound for the achievable sum rate that is met with equality in several scenarios.

Lemma 1. For every $\mathbf{K}_{w}$ satisfying (4), the sum rate of multiple description with two levels of receivers satisfies

$$
\sum_{l=1}^{L} R_{l} \geq \frac{L}{k\left(\begin{array}{l}
L \\
k
\end{array}\right)}\left[\sum_{|S|=k} h\left(\mathbf{u}_{S}\right)\right]-h\left(\mathbf{u}_{1}, \ldots, \mathbf{u}_{L} \mid \mathbf{x}\right) .
$$

Proof. Let $T=\{1, \ldots, L\}$ in (5) and we get

$$
\sum_{l=1}^{L} R_{l}^{\prime} \geq\left[\sum_{l=1}^{L} h\left(\mathbf{u}_{l}\right)\right]-h\left(\mathbf{u}_{1}, \ldots, \mathbf{u}_{L} \mid \mathbf{x}\right)
$$

Summing over all the inequalities in (7) and we get

$$
\sum_{S:|S|=k} \sum_{l \in S}\left(R_{l}^{\prime}-R_{l}\right) \leq\left[\sum_{S:|S|=k} \sum_{l \in S} h\left(\mathbf{u}_{l}\right)\right]-\sum_{S:|S|=k} h\left(\mathbf{u}_{S}\right) .
$$

A simple counting argument leads us to

$$
\sum_{l=1}^{L}\left(R_{l}^{\prime}-R_{l}\right) \leq\left[\sum_{l=1}^{L} h\left(\mathbf{u}_{l}\right)\right]-\frac{1}{\left(\begin{array}{l}
L-1 \\
k-1
\end{array}\right)} \sum_{S,|S|=k} h\left(\mathbf{u}_{S}\right) .
$$

Subtracting (13) from (11) and using the identity

$$
L\left(\begin{array}{l}
L-1 \\
k-1
\end{array}\right)=k\left(\begin{array}{l}
L \\
k
\end{array}\right)
$$

we get the desired result.

Observe that this is only a lower bound on the sum rate. There are many ways of combining equations (5) and (7) and as such, the bound in (10) may not be achievable. However, there are some important cases where (10) is indeed achievable. When $k=1$, it is shown in [11] that (10) is achievable by relying on the combinatorial structure of the achievable region: specifically, the region was shown to be a contra-polymatroid. Another example for $k>1$ is when all the distortions in the first layer are equal. We have the following result. 
Lemma 2. When all the distortion constraints in the first layer are equal, if we choose u's such that all the $h\left(\mathbf{u}_{l}\right)$ are equal for $l=1, \ldots, L$ and all the $h\left(\mathbf{u}_{S}\right)$ are equal for $\forall S \subset\{1, \ldots L\},|S|=k$, then (10) is achievable.

Proof. In this case, by the symmetry of the problem we can choose all the $R_{l}^{\prime}$ 's to be equal $R^{\prime}$ and all the $R_{l}$ 's to be equal to $R$. Thus all the constraints on in (5) can be simplified to

$$
L R^{\prime} \geq L h\left(\mathbf{u}_{l}\right)-h\left(\mathbf{u}_{1}, \ldots, \mathbf{u}_{L} \mid \mathbf{x}\right) .
$$

and (7) can be simplified to

$$
k\left(R^{\prime}-R\right) \leq k h\left(\mathbf{u}_{l}\right)-h\left(\mathbf{u}_{S}\right), \quad \forall S \subset\{1, \ldots L\},|S|=k .
$$

Thus we have

$$
R \geq \frac{1}{k} h\left(\mathbf{u}_{S}\right)-\frac{1}{L} h\left(\mathbf{u}_{1}, \ldots, \mathbf{u}_{L} \mid \mathbf{x}\right)
$$

which is equivalent to (10) under the condition stated in this lemma.

Later we will show that under the symmetric distortion constraints, we can restrict ourself to consider that the u's such that all the $h\left(\mathbf{u}_{l}\right)$ are equal for $l=1, \ldots, L$ and all the $h\left(\mathbf{u}_{S}\right)$ are equal for $\forall S \subset\{1, \ldots L\},|S|=k$, and still achieve the optimal sum rate. We call this choice of u's as symmetric descriptions.

With asymmetric distortion constraints in the first layer, we have the following result.

Lemma 3. With $k=2$ and $L=3$, (10) is achievable.

Proof. From the proof of Lemma 1, it is clear that we only need to show that there exists $R_{1}^{\prime}, R_{2}^{\prime}$ and $R_{3}^{\prime}$ satisfying (5) and nonnegative $R_{1}, R_{2}, R_{3}$ such that

$$
R_{1}^{\prime}+R_{2}^{\prime}+R_{3}^{\prime}=h\left(\mathbf{u}_{1}\right)+h\left(\mathbf{u}_{2}\right)+h\left(\mathbf{u}_{3}\right)-h\left(\mathbf{u}_{1}, \mathbf{u}_{2}, \mathbf{u}_{3} \mid \mathbf{x}\right),
$$

and

$$
\sum_{|S|=2, l \in S}\left(R_{l}^{\prime}-R_{l}\right)=\left[\sum_{|S|=2, l \in S} h\left(\mathbf{u}_{l}\right)\right]-h\left(\mathbf{u}_{l}, l \in S\right) .
$$

Solving the previous two equations, we have

$$
\begin{aligned}
& R_{1}=R_{1}^{\prime}-\left[h\left(\mathbf{u}_{1}\right)+h\left(\mathbf{u}_{2}, \mathbf{u}_{3}\right)-\frac{1}{2}\left(h\left(\mathbf{u}_{1}, \mathbf{u}_{2}\right)+h\left(\mathbf{u}_{2}, \mathbf{u}_{3}\right)+h\left(\mathbf{u}_{1}, \mathbf{u}_{3}\right)\right)\right], \\
& R_{2}=R_{2}^{\prime}-\left[h\left(\mathbf{u}_{2}\right)+h\left(\mathbf{u}_{1}, \mathbf{u}_{3}\right)-\frac{1}{2}\left(h\left(\mathbf{u}_{1}, \mathbf{u}_{2}\right)+h\left(\mathbf{u}_{2}, \mathbf{u}_{3}\right)+h\left(\mathbf{u}_{1}, \mathbf{u}_{3}\right)\right)\right], \\
& R_{3}=R_{3}^{\prime}-\left[h\left(\mathbf{u}_{3}\right)+h\left(\mathbf{u}_{1}, \mathbf{u}_{2}\right)-\frac{1}{2}\left(h\left(\mathbf{u}_{1}, \mathbf{u}_{2}\right)+h\left(\mathbf{u}_{2}, \mathbf{u}_{3}\right)+h\left(\mathbf{u}_{1}, \mathbf{u}_{3}\right)\right)\right] .
\end{aligned}
$$


Suppose we choose $R_{1 a}^{\prime}, R_{2 a}^{\prime}$ and $R_{3 a}^{\prime}$ satisfying (5) and (17). We have

$$
\begin{aligned}
R_{1}+R_{2} & =R_{1 a}^{\prime}+R_{2 a}^{\prime}-\left[h\left(\mathbf{u}_{1}\right)+h\left(\mathbf{u}_{2}\right)-h\left(\mathbf{u}_{1}, \mathbf{u}_{2}\right)\right] \\
& \geq\left[h\left(\mathbf{u}_{1}\right)+h\left(\mathbf{u}_{2}\right)-h\left(\mathbf{u}_{1}, \mathbf{u}_{2} \mid \mathbf{x}\right)\right]-\left[h\left(\mathbf{u}_{1}\right)+h\left(\mathbf{u}_{2}\right)-h\left(\mathbf{u}_{1}, \mathbf{u}_{2}\right)\right] \\
& \geq 0
\end{aligned}
$$

Similarly, we have $R_{2}+R_{3} \geq 0$ and $R_{1}+R_{3} \geq 0$. Hence at most one of $R_{1}, R_{2}$ and $R_{3}$ can be negative. If all of them are nonnegative, our proof is complete. On the other hand, suppose one of them is negative for our choice of $R_{1 a}^{\prime}, R_{2 a}^{\prime}$ and $R_{3 a}^{\prime}$. We next show that we can find $R_{1 b}^{\prime}, R_{2 b}^{\prime}$ and $R_{3 b}^{\prime}$ satisfying (5) and (17) and resulting in nonnegative $R_{1}, R_{2}$ and $R_{3}$. Without loss of generality, suppose $R_{3}<0$. Then we can reduce $R_{1 a}^{\prime}$ to $R_{1 b}^{\prime}$ and $R_{2 a}^{\prime}$ to $R_{2 b}^{\prime}$ so that $R_{1} \geq 0, R_{2} \geq 0$, and

$$
R_{1 b}^{\prime}+R_{2 b}^{\prime}=h\left(\mathbf{u}_{1}\right)+h\left(\mathbf{u}_{2}\right)-h\left(\mathbf{u}_{1}, \mathbf{u}_{2} \mid \mathbf{x}\right),
$$

and hence

$$
R_{3 b}^{\prime}=h\left(\mathbf{u}_{3}\right)+h\left(\mathbf{u}_{1}, \mathbf{u}_{2} \mid \mathbf{x}\right)-h\left(\mathbf{u}_{1}, \mathbf{u}_{2}, \mathbf{u}_{3} \mid \mathbf{x}\right) .
$$

Then

$$
\begin{aligned}
R_{3}= & h\left(\mathbf{u}_{3}\right)+h\left(\mathbf{u}_{1}, \mathbf{u}_{2} \mid \mathbf{x}\right)-h\left(\mathbf{u}_{1}, \mathbf{u}_{2}, \mathbf{u}_{3} \mid \mathbf{x}\right) \\
& -\left[h\left(\mathbf{u}_{3}\right)+h\left(\mathbf{u}_{1}, \mathbf{u}_{2}\right)-\frac{1}{2}\left(h\left(\mathbf{u}_{1}, \mathbf{u}_{2}\right)+h\left(\mathbf{u}_{2}, \mathbf{u}_{3}\right)+h\left(\mathbf{u}_{1}, \mathbf{u}_{3}\right)\right)\right] \\
= & h\left(\mathbf{u}_{1}, \mathbf{u}_{2} \mid \mathbf{x}\right)-h\left(\mathbf{u}_{1}, \mathbf{u}_{2}\right)+\frac{1}{2}\left(h\left(\mathbf{u}_{1}, \mathbf{u}_{2}\right)+h\left(\mathbf{u}_{2}, \mathbf{u}_{3}\right)+h\left(\mathbf{u}_{1}, \mathbf{u}_{3}\right)\right)-h\left(\mathbf{u}_{1}, \mathbf{u}_{2}, \mathbf{u}_{3} \mid \mathbf{x}\right) \\
\geq & h\left(\mathbf{u}_{1}, \mathbf{u}_{2} \mid \mathbf{x}\right)-h\left(\mathbf{u}_{1}, \mathbf{u}_{2}\right)+h\left(\mathbf{u}_{1}, \mathbf{u}_{2}, \mathbf{u}_{3}\right)-h\left(\mathbf{u}_{1}, \mathbf{u}_{2}, \mathbf{u}_{3} \mid \mathbf{x}\right) \\
= & I\left(\mathbf{u}_{1}, \mathbf{u}_{2}, \mathbf{u}_{3} ; \mathbf{x}\right)-I\left(\mathbf{u}_{1}, \mathbf{u}_{2} ; \mathbf{x}\right) \\
\geq & 0
\end{aligned}
$$

\section{Lower Bound to Sum Rate}

Our main result is the following lower bound on the sum rate of any multiple descriptions that meet the given distortion constraints with two levels of receivers.

Theorem 1. Meeting the distortion constraints $\left(\mathbf{D}_{S}, \mathbf{D}_{L}\right)$, the sum rate of any multiple description scheme is lower bounded by

$$
\sum_{l=1}^{L} R_{l} \geq \frac{L}{k\left(\begin{array}{l}
L \\
k
\end{array}\right)} \sum_{S:|S|=k}\left[\frac{1}{2} \log \frac{\left|\mathbf{K}_{x}+\mathbf{K}_{z}\right|}{\left|\mathbf{D}_{S}+\mathbf{K}_{z}\right|}\right]+\frac{1}{2} \log \frac{\left|\mathbf{K}_{x}\right|}{\left|\mathbf{K}_{x}+\mathbf{K}_{z}\right|}+\frac{1}{2} \log \frac{\left|\mathbf{D}_{L}+\mathbf{K}_{z}\right|}{\left|\mathbf{D}_{L}\right|}
$$


Proof. Define a memoryless Gaussian process $\{\mathbf{z}[m]\}_{m=1}^{n}$ with marginal distribution $\mathcal{N}\left(0, \mathbf{K}_{z}\right)$. We let $\{\mathbf{z}[m]\}_{m=1}^{n}$ be independent of the information source $\mathbf{x}^{n}$ as well as the codebooks $C_{l}$ for $l=1, \ldots, L$. Construct a random process $\mathbf{y}^{n}=(\mathbf{y}[1], \ldots, \mathbf{y}[n])$ by

$$
\mathbf{y}[m]=\mathbf{x}[m]+\mathbf{z}[m], \quad m=1, \ldots, n .
$$

It follows that $\{\mathbf{y}[m]\}$ is also a memoryless Gaussian process with marginal distribution $\mathcal{N}\left(0, \mathbf{K}_{y}\right)$; here $\mathbf{K}_{y}=\mathbf{K}_{x}+\mathbf{K}_{z}$.

Consider the following sequence of lower bounds to the sum rate of the multiple descriptions:

$$
\begin{aligned}
n L R \geq & \sum_{l=1}^{L} H\left(C_{l}\right)=\left[\sum_{l=1}^{L} H\left(C_{l}\right)\right]-H\left(C_{1}, \ldots, C_{L} \mid \mathbf{x}^{n}\right) \\
& \stackrel{(a)}{\geq} \frac{L}{k\left(\begin{array}{l}
L \\
k
\end{array}\right)}\left[\sum_{|S|=k} H\left(C_{S}\right)\right]-H\left(C_{1}, \ldots, C_{L} \mid \mathbf{x}^{n}\right) \\
\geq & \frac{L}{k\left(\begin{array}{l}
L \\
k
\end{array}\right)}\left[\sum_{|S|=k} H\left(C_{S}\right)\right]-H\left(C_{1}, \ldots, C_{L} \mid \mathbf{x}^{n}\right)+H\left(C_{1}, \ldots, C_{L}\right)-H\left(C_{1}, \ldots, C_{L}\right) \\
& -\left(\frac{L}{k\left(\begin{array}{l}
L \\
k
\end{array}\right)}\left[\sum_{|S|=k} H\left(C_{S} \mid \mathbf{y}^{n}\right)\right]-H\left(C_{1}, \ldots, C_{L} \mid \mathbf{y}^{n}\right)\right) \\
= & \frac{L}{k\left(\begin{array}{l}
L \\
k
\end{array}\right)}\left[\sum_{|S|=k} I\left(C_{S} ; \mathbf{y}^{n}\right)\right]+I\left(C_{1}, \ldots, C_{L} ; \mathbf{x}^{n}\right)-I\left(C_{1}, \ldots, C_{L} ; \mathbf{y}^{n}\right) \\
= & \frac{L}{k\left(\begin{array}{l}
L \\
k
\end{array}\right)} \sum_{|S|=k}\left[h\left(\mathbf{y}^{n}\right)-h\left(\mathbf{y}^{n} \mid C_{S}\right)\right]+h\left(\mathbf{x}^{n}\right)-h\left(\mathbf{y}^{n}\right) \\
& +h\left(\mathbf{y}^{n} \mid C_{1}, \ldots, C_{L}\right)-h\left(\mathbf{x}^{n} \mid C_{1}, \ldots, C_{L}\right),
\end{aligned}
$$

where in steps (a) and (b) we used the inequality related to the entropy rate of subsets [4, Theorem 16.5.1].

We know that [4, Theorem 9.4.1]

$$
\begin{aligned}
h\left(\mathbf{x}^{n}\right) & =\frac{1}{2} \log (2 \pi e)^{N n}\left|\mathbf{K}_{x}\right|^{n}, \\
h\left(\mathbf{y}^{n}\right) & =\frac{1}{2} \log (2 \pi e)^{N n}\left|\mathbf{K}_{y}\right|^{n}=\frac{1}{2} \log (2 \pi e)^{N n}\left|\mathbf{K}_{x}+\mathbf{K}_{z}\right|^{n} .
\end{aligned}
$$

We also have an upper bound on $h\left(\mathbf{y}^{n} \mid C_{S}\right)$ for $\forall S \subset\{1, \ldots, L\},|S|=k$ based on the distortion 
constraints of the first layer decoder:

$$
\begin{aligned}
h\left(\mathbf{y}^{n} \mid C_{S}\right) & \leq \sum_{m=1}^{n} h\left(\mathbf{y}[m] \mid C_{S}\right) \\
& \leq \sum_{m=1}^{n} \frac{1}{2} \log (2 \pi e)^{N}\left|\operatorname{Cov}\left[\mathbf{y}[m] \mid C_{S}\right]\right| \\
& \leq \frac{1}{2} \log (2 \pi e)^{N n}+\frac{n}{2} \log \left|\frac{1}{n} \sum_{m=1}^{n} \operatorname{Cov}\left[\mathbf{y}[m] \mid C_{S}\right]\right| \\
& =\frac{1}{2} \log (2 \pi e)^{N n}+\frac{n}{2} \log \left|\frac{1}{n} \sum_{m=1}^{n} \operatorname{Cov}\left[(\mathbf{x}[m]+\mathbf{z}[m]) \mid C_{S}\right]\right| \\
& =\frac{1}{2} \log (2 \pi e)^{N n}+\frac{n}{2} \log \left|\frac{1}{n} \sum_{m=1}^{n} \operatorname{Cov}\left[\mathbf{x}[m] \mid C_{S}\right]+\mathbf{K}_{z}\right| \\
& \leq \frac{1}{2} \log (2 \pi e)^{N n}+\frac{n}{2} \log \left|\mathbf{D}_{S}+\mathbf{K}_{z}\right| \\
& =\frac{1}{2} \log (2 \pi e)^{N n}\left|\mathbf{D}_{S}+\mathbf{K}_{z}\right|^{n} .
\end{aligned}
$$

Next we bound the difference of the last two terms of (25).

$$
\begin{aligned}
h\left(\mathbf{y}^{n} \mid C_{1},\right. & \left.\ldots, C_{L}\right)-h\left(\mathbf{x}^{n} \mid C_{1}, \ldots, C_{L}\right) \\
& =h\left(\mathbf{y}^{n} \mid C_{1}, \ldots, C_{L}\right)-h\left(\mathbf{x}^{n} \mid \mathbf{z}^{n}, C_{1}, \ldots, C_{L}\right) \\
& =h\left(\mathbf{y}^{n} \mid C_{1}, \ldots, C_{L}\right)-h\left(\mathbf{y}^{n} \mid \mathbf{z}^{n}, C_{1}, \ldots, C_{L}\right) \\
& =I\left(\mathbf{y}^{n} ; \mathbf{z}^{n} \mid C_{1}, \ldots, C_{L}\right) .
\end{aligned}
$$

Letting

$$
\mathbf{K}_{c}[m] \stackrel{\text { def }}{=} \operatorname{Cov}\left[\mathbf{x}[m]-\hat{\mathbf{x}}_{L}[m]\right]
$$


we have

$$
\begin{aligned}
I\left(\mathbf{y}^{n} ; \mathbf{z}^{n} \mid C_{1}, \ldots, C_{L}\right) & =h\left(\mathbf{z}^{n} \mid C_{1}, \ldots, C_{L}\right)-h\left(\mathbf{z}^{n} \mid \mathbf{y}^{n}, C_{1}, \ldots, C_{L}\right) \\
& =h\left(\mathbf{z}^{n}\right)-h\left(\mathbf{z}^{n} \mid \mathbf{y}^{n}-\hat{\mathbf{x}}_{L}^{n}, C_{1}, \ldots, C_{L}\right) \\
& \geq h\left(\mathbf{z}^{n}\right)-h\left(\mathbf{z}^{n} \mid \mathbf{y}^{n}-\hat{\mathbf{x}}_{L}^{n}\right) \\
& =\sum_{m=1}^{n}\left(h(\mathbf{z}[m])-h\left(\mathbf{z}[m] \mid \mathbf{z}[1], \ldots, \mathbf{z}[m-1], \mathbf{y}^{n}-\hat{\mathbf{x}}_{L}^{n}\right)\right) \\
& \geq \sum_{m=1}^{n}\left(h(\mathbf{z}[m])-h\left(\mathbf{z}[m] \mid \mathbf{y}[m]-\hat{\mathbf{x}}_{0}[m]\right)\right) \\
& =\sum_{m=1}^{n} I\left(\mathbf{z}[m] ; \mathbf{x}[m]-\hat{\mathbf{x}}_{L}[m]+\mathbf{z}[m]\right) \\
& \left(\frac{a)}{\geq} \sum_{m=1}^{n} \frac{1}{2} \log \frac{\left|\mathbf{K}_{c}[m]+\mathbf{K}_{z}[m]\right|}{\left|\mathbf{K}_{c}[m]\right|}\right. \\
& \stackrel{(b)}{\geq} \frac{n}{2} \log \frac{\left|\mathbf{D}_{L}+\mathbf{K}_{z}\right|}{\left|\mathbf{D}_{L}\right|}
\end{aligned}
$$

where (a) is from (29) and the worst case noise result [9, Lemma II.2]. The justification for (b) is from the convexity of $\log \frac{|\mathbf{A}+\mathbf{B}|}{|\mathbf{A}|}$ in $\mathbf{A}$ and (2). From (28) and (30) we have

$$
h\left(\mathbf{y}^{n} \mid C_{1}, \ldots, C_{L}\right)-h\left(\mathbf{x}^{n} \mid C_{1}, \ldots, C_{L}\right) \geq \frac{n}{2} \log \frac{\left|\mathbf{D}_{L}+\mathbf{K}_{z}\right|}{\left|\mathbf{D}_{L}\right|} .
$$

Combining (25), (26), (27) and (31), and taking the supremum over all positive definite matrices $\mathbf{K}_{z}$, we have proved the claimed inequality.

As in the case of the necessary condition for achievable sum rate, we can use different combinations of entropy rate subset in the previous proof and hence give different outer bounds. This complication arises because there are many subsets of size $k$. Nevertheless, we show in the next section that when (10) is achievable for the optimal Gaussian multiple description scheme, the lower bound in (24) is actually tight.

\section{Optimal Sum Rate}

In this section we provide conditions under which the lower bound (24) is achieved by the separation architecture in Figure 2 and is hence tight. 
Theorem 2. If there exists a choice of $\mathbf{K}_{w}$ of the form

$$
\mathbf{K}_{w}=\left(\begin{array}{ccccc}
\mathbf{K}_{w_{1}} & -\mathbf{A} & -\mathbf{A} & \ldots & -\mathbf{A} \\
-\mathbf{A} & \mathbf{K}_{w_{2}} & -\mathbf{A} & \ldots & -\mathbf{A} \\
\ldots \ldots & \ldots & \ldots & \ldots & \ldots \\
-\mathbf{A} & \ldots & -\mathbf{A} & \mathbf{K}_{w_{L-1}} & -\mathbf{A} \\
-\mathbf{A} & \ldots & -\mathbf{A} & -\mathbf{A} & \mathbf{K}_{w_{L}}
\end{array}\right)
$$

where $\mathbf{0} \prec \mathbf{A} \prec \mathbf{K}_{x}$, such that (10) is achievable and all the distortion constraints are met with equality, then the optimal sum rate is given by (24).

Proof. To compare the lower bound (24) and the achievable sum rate (10), one way is to directly calculate the optimal value of these two bounds. While this approach is reasonable for the scalar Gaussian source case, it is quite involved to carry out this program for the vector Gaussian source case we are studying. In the following, we provide an alternative characterization of the achievable rate; this makes the way for a much easier to comparison with the lower bound (41).

Consider an $\mathcal{N}\left(0, \mathbf{K}_{z}\right)$ Gaussian random vector $\mathbf{z}$ that is independent of $\mathbf{x}$ and all $\mathbf{w}_{l}$ 's. Define $\mathbf{y}=\mathbf{x}+\mathbf{z}$. The following rates are achievable using the separation architecture of Figure 2:

$$
\begin{aligned}
\sum_{l=1}^{L} R_{l}= & \frac{L}{k\left(\begin{array}{l}
L \\
k
\end{array}\right)} \sum_{|S|=k} h\left(\mathbf{u}_{S}\right)-h\left(\mathbf{u}_{1}, \ldots, \mathbf{u}_{L} \mid \mathbf{x}\right) \\
= & \frac{L}{k\left(\begin{array}{l}
L \\
k
\end{array}\right)} \sum_{|S|=k} h\left(\mathbf{u}_{S}\right)-h\left(\mathbf{u}_{1}, \ldots, \mathbf{u}_{L} \mid \mathbf{x}\right)+h\left(\mathbf{u}_{1}, \ldots, \mathbf{u}_{L}\right)-h\left(\mathbf{u}_{1}, \ldots, \mathbf{u}_{L}\right) \\
& \stackrel{(a)}{\geq} \frac{L}{k\left(\begin{array}{l}
L \\
k
\end{array}\right)} \sum_{|S|=k} h\left(\mathbf{u}_{S}\right)-h\left(\mathbf{u}_{1}, \ldots, \mathbf{u}_{L} \mid \mathbf{x}\right)+h\left(\mathbf{u}_{1}, \ldots, \mathbf{u}_{L}\right)-h\left(\mathbf{u}_{1}, \ldots, \mathbf{u}_{L}\right) \\
& -\left(\frac{L}{k\left(\begin{array}{l}
L \\
k
\end{array}\right)} \sum_{|S|=k} h\left(\mathbf{u}_{S} \mid \mathbf{y}\right)-h\left(\mathbf{u}_{1}, \ldots, \mathbf{u}_{L} \mid \mathbf{y}\right)\right) \\
= & \frac{L}{k\left(\begin{array}{l}
L \\
k
\end{array}\right)} \sum_{|S|=k} I\left(\mathbf{u}_{S} ; \mathbf{y}\right)+I\left(\mathbf{u}_{1}, \ldots, \mathbf{u}_{L} ; \mathbf{x}^{n}\right)-I\left(\mathbf{u}_{1}, \ldots, \mathbf{u}_{L} ; \mathbf{y}\right) \\
= & \frac{L}{k\left(\begin{array}{l}
L \\
k
\end{array}\right)} \sum_{|S|=k}\left(h(\mathbf{y})-h\left(\mathbf{y} \mid \mathbf{u}_{S}\right)\right)+(h(\mathbf{x})-h(\mathbf{y}))+\left(h\left(\mathbf{y} \mid \mathbf{u}_{1}, \ldots, \mathbf{u}_{L}\right)-h\left(\mathbf{x} \mid \mathbf{u}_{1}, \ldots, \mathbf{u}_{L}\right)\right) \\
= & \frac{L}{k\left(\begin{array}{l}
L \\
k
\end{array}\right)} \sum_{|S|=k}\left[\frac{1}{2} \log \frac{\left|\mathbf{K}_{x}+\mathbf{K}_{z}\right|}{\left|\operatorname{Cov}\left[\mathbf{x} \mid \mathbf{u}_{S}\right]+\mathbf{K}_{z}\right|}\right]+\frac{1}{2} \log \frac{\left|\mathbf{K}_{x}\right|}{\left|\mathbf{K}_{x}+\mathbf{K}_{z}\right|}+\frac{1}{2} \log \frac{\left|\operatorname{Cov}\left[\mathbf{x} \mid \mathbf{u}_{1}, \ldots, \mathbf{u}_{L}\right]+\mathbf{K}_{z}\right|}{|| \operatorname{Cov}\left[\mathbf{x} \mid \mathbf{u}_{1}, \ldots, \mathbf{u}_{L}\right] \mid}
\end{aligned}
$$

where the last step follows from a procedural Gaussian MMSE calculation. 
Note that if

$$
\frac{1}{k\left(\begin{array}{l}
L \\
k
\end{array}\right)} \sum_{|S|=k} h\left(\mathbf{u}_{S} \mid \mathbf{y}\right)-\frac{1}{L} h\left(\mathbf{u}_{1}, \ldots, \mathbf{u}_{L} \mid \mathbf{y}\right)=0,
$$

then (a) in (33) is actually an equality. Thus, if our choices of $\mathbf{K}_{w}$ and $\mathbf{K}_{z}$ satisfy the following two conditions:

- the equation (34) is true.

- the distortion constraints are satisfied with equality, i.e.,

$$
\begin{aligned}
& \operatorname{Cov}\left[\mathbf{x} \mid \mathbf{u}_{S}\right]=\mathbf{D}_{S}, \quad \forall S \subset\{1, \ldots, L\},|S|=k, \\
& \operatorname{Cov}\left[\mathbf{x} \mid \mathbf{u}_{1}, \ldots, \mathbf{u}_{L}\right]=\mathbf{D}_{L},
\end{aligned}
$$

then the achievable rate (33) matches the lower bound (24), and thus we characterized the optimal sum rate. In the following, we examine under what circumstances the above two conditions are true.

First, it is easy to check that the following sufficient condition for (34) is true.

Proposition 1. Let $\mathbf{w}_{1}, \cdots, \mathbf{w}_{L}$ be zero-mean jointly Gaussian random vectors independent of $\mathbf{x}$, and let $\mathbf{K}_{w}$ indicates the positive definite covariance matrix for $\left(\mathbf{w}_{1}, \cdots, \mathbf{w}_{L}\right)$. Let

$$
\mathbf{u}_{l}=\mathbf{x}+\mathbf{w}_{l}, \quad l=1, \ldots, L .
$$

If $\mathbf{K}_{w}$ takes form of (32) with $\mathbf{0} \prec \mathbf{A} \prec \mathbf{K}_{x}$, then there exists $\mathbf{y}=\mathbf{x}+\mathbf{z}$ such that (34) is true, where $\mathbf{z}$ is an $\mathcal{N}\left(0, \mathbf{K}_{z}\right)$ Gaussian random vector that is independent of $\mathbf{x}$ and all $\mathbf{w}_{l}$ 's, and the covariance matrix of $\mathbf{z}$ is $\mathbf{K}_{z}=\mathbf{K}_{x}\left(\mathbf{K}_{x}-\mathbf{A}\right)^{-1} \mathbf{K}_{x}-\mathbf{K}_{x}$.

\section{Proof. See Appendix A.}

Next we characterize the $\mathbf{A}$ in (32) from the condition that all distortion constraints are met with equality. From the distortion constraints of the first level of receivers, we have that for all $S \in\{1,2, \cdots, L\},|S|=k$,

$$
\left[\left(\mathbf{D}_{S}^{-1}-\mathbf{K}_{x}^{-1}\right)^{-1}+\mathbf{A}\right]^{-1}=\sum_{l \in S}\left[\mathbf{K}_{w_{l}}+\mathbf{A}\right]^{-1}
$$

From the distortion constraint of the second level of receiver, we have

$$
\left[\left(\mathbf{D}_{L}^{-1}-\mathbf{K}_{x}^{-1}\right)^{-1}+\mathbf{A}\right]^{-1}=\sum_{l=1}^{L}\left[\mathbf{K}_{w_{l}}+\mathbf{A}\right]^{-1}
$$


Summing up the equalities represented in (36) for each subset $S$, we have

$$
\sum_{S:|S|=k}\left[\left(\mathbf{D}_{S}^{-1}-\mathbf{K}_{x}^{-1}\right)^{-1}+\mathbf{A}\right]^{-1}=\frac{k}{L}\left(\begin{array}{l}
L \\
k
\end{array}\right) \sum_{l=1}^{L}\left[\mathbf{K}_{w_{l}}+\mathbf{A}\right]^{-1}=\frac{k}{L}\left(\begin{array}{l}
L \\
k
\end{array}\right)\left[\left(\mathbf{D}_{L}^{-1}-\mathbf{K}_{x}^{-1}\right)^{-1}+\mathbf{A}\right]^{-1} \text {. }
$$

Define

$$
\begin{aligned}
& \boldsymbol{\Lambda}_{S}=\left(\mathbf{D}_{S}^{-1}-\mathbf{K}_{x}^{-1}\right)^{-1}, \quad \forall S \in\{1,2, \cdots, L\},|S|=k, \\
& \boldsymbol{\Lambda}_{L}=\left(\mathbf{D}_{L}^{-1}-\mathbf{K}_{x}^{-1}\right)^{-1} .
\end{aligned}
$$

We can rewrite (38) as

$$
\sum_{S,|S|=k}\left[\boldsymbol{\Lambda}_{S}+\mathbf{A}\right]^{-1}=\frac{k}{L}\left(\begin{array}{l}
L \\
k
\end{array}\right)\left[\boldsymbol{\Lambda}_{L}+\mathbf{A}\right]^{-1}
$$

Thus, when all the distortion constraints are met with equality, we can solve A from (40). From [11, Lemma 5], we know that if the solution $\mathbf{A}$ is positive definite and (37) holds, then the covariance matrix $\mathbf{K}_{w}$ defined in (32) is positive definite.

To summarize, we have the following theorem.

Theorem 3. Given distortion constraints $\left(\mathbf{D}_{S}, \mathbf{D}_{L}\right)$. If there exists a solution $\mathbf{A}^{*}$ to (40) and $\mathbf{0} \prec \mathbf{A}^{*} \prec \mathbf{K}_{x}$, such that (10) is achievable and all the distortion constraints are met with equality (i.e., (36) and (37) hold), then the analog-digital separation architecture with $\mathbf{K}_{w}$ defined in (32) with $\mathbf{A}=\mathbf{A}^{*}$ achieves the optimal sum rate, and the optimal $\mathbf{K}_{z}$ for lower bound (24) is $\mathbf{K}_{z}=$ $\mathbf{K}_{x}\left(\mathbf{K}_{x}-\mathbf{A}^{*}\right)^{-1} \mathbf{K}_{x}-\mathbf{K}_{x}$.

From Theorem 3 we know that the analog-digital separation architecture achieves the optimal sum rate if the given distortion constraints $\left(\mathbf{D}_{S}, \mathbf{D}_{L}\right)$ satisfy the condition for Theorem 3 , and we can calculate the optimal $\mathbf{K}_{w}$ by solving matrix equations. In general, not all the distortion constraints may be achieved by equality. In this case, we may be able to show that there exists a analog-digital separation architecture that achieves the sum rate lower bound, and results in distortions $\left(\mathbf{D}_{S}^{*}, \mathbf{D}_{L}^{*}\right)$ such that $\mathbf{D}_{S}^{*} \preccurlyeq \mathbf{D}_{S}$ for $\forall S \in\{1,2, \cdots, L\},|S|=k$, and $\mathbf{D}_{L}^{*} \preccurlyeq \mathbf{D}_{L}$.

When $k=1$, it is shown in [11] that (10) is achievable due to the contra-polymatroid structure of the achievable region. It is also shown in [11] that the problem of the distortion constraints not being met with equality can be handled through a technique called enhancing. In the following sections, we provide instance of the multiple description problem with $k>1$ where the optimal sum rate can still be characterized. 


\section{Symmetric Distortion Constraints}

A natural instance of the multiple description problem imposes a distortion constraint based on the cardinality of the subset of descriptions received by the user (as opposed to requiring different distortion constraints for specific subset of the descriptions being received). In the problem of our focus, this means that we have only two distortion constraints $\mathbf{D}_{k}$ and $\mathbf{D}_{L}$ : either corresponding to $k$ or all of the descriptions being received. From the symmetry of the problem, we have seen in Lemma 2 that to achieve the optimal sum rate we can use the same rate for all $L$ encoders, and further that, the sum rate given in (10) is achievable if we use symmetric descriptions. The only complication left is that not all of the distortion constraints may be met with equality. We now work to address this issue and show that the separation architecture in Figure 2 achieves the sum rate lower bound given in Theorem 1 .

Specifically, we have the following corollary of Theorem 1 by specializing all the $\mathbf{D}_{S}$ to be $\mathbf{D}_{k}$ in (24).

Theorem 4. For given symmetric distortion constraints $\left(\mathbf{D}_{k}, \mathbf{D}_{L}\right)$, the optimal sum rate for multiple descriptions of a memoryless $\mathcal{N}\left(0, \mathbf{K}_{x}\right)$ Gaussian source is

$$
\frac{L}{k\left(\begin{array}{l}
L \\
k
\end{array}\right)}\left[\frac{1}{2} \log \frac{\left|\mathbf{K}_{x}+\mathbf{K}_{z}\right|}{\left|\mathbf{D}_{k}+\mathbf{K}_{z}\right|}\right]+\frac{1}{2} \log \frac{\left|\mathbf{K}_{x}\right|}{\left|\mathbf{K}_{x}+\mathbf{K}_{z}\right|}+\frac{1}{2} \log \frac{\left|\mathbf{D}_{L}+\mathbf{K}_{z}\right|}{\left|\mathbf{D}_{L}\right|} .
$$

In the following we first consider a scalar Gaussian source. Using the insights developed, the proof for the general vector case is more transparent.

\subsection{Scalar Gaussian Source}

Consider a scalar memoryless Gaussian information source; the marginal distribution is $\mathcal{N}\left(0, \sigma_{x}^{2}\right)$. Let the distortion constraints be $\left(d_{k}, d_{L}\right)$, with the natural ordering

$$
0<d_{L}<d_{k}<\sigma_{x}^{2}
$$

As an achievable scheme, we have the natural separation architecture from Figure 2. Specifically, let the choice of the parameter (covariance matrix for $\left(w_{1}, \ldots, w_{L}\right)$ ) be

$$
\mathbf{K}_{w}=\left(\begin{array}{ccccc}
\sigma^{2} & -a & -a & \ldots & -a \\
-a & \sigma^{2} & -a & \ldots & -a \\
\ldots & \ldots & \ldots & \ldots & \ldots \\
-a & \ldots & -a & \sigma^{2} & -a \\
-a & \ldots & -a & -a & \sigma^{2}
\end{array}\right)
$$


Setting to equality the distortion conditions ((36) and (37)), we see that

$$
\begin{aligned}
\sigma^{2} & =\frac{(L k-k)\left[d_{k}^{-1}-\sigma_{x}^{-2}\right]^{-1}-(L k-L)\left[d_{L}^{-1}-\sigma_{x}^{-2}\right]^{-1}}{L-k}, \\
a & =\frac{k\left[d_{k}^{-1}-\sigma_{x}^{-2}\right]^{-1}-L\left[d_{L}^{-1}-\sigma_{x}^{-2}\right]^{-1}}{L-k}
\end{aligned}
$$

We can now check whether the condition for Theorem 3, i.e., $0<a<\sigma_{x}^{2}$, is true or not. We do this by separately considering three cases.

Case 1: $0<\frac{k\left[d_{k}^{-1}-\sigma_{x}^{-2}\right]^{-1}-L\left[d_{L}^{-1}-\sigma_{x}^{-2}\right]^{-1}}{L-k}<\sigma_{x}^{2}$.

In this case, the condition $0<a<\sigma_{x}^{2}$ holds. From Theorem 3, we conclude that the separation architecture achieves the optimal symmetric rate; specifically, the covariance matrix of $\left(w_{1}, \ldots, w_{L}\right)$ takes the form (42) with $a$ and $\sigma^{2}$ as given by (43).

Case 2: $\quad \frac{k\left[d_{k}^{-1}-\sigma_{x}^{-2}\right]^{-1}-L\left[d_{L}^{-1}-\sigma_{x}^{-2}\right]^{-1}}{L-k} \geq \sigma_{x}^{2}$.

In this case, the condition $0<a<\sigma_{x}^{2}$ does not hold. However, the analog-digital separation architecture can still achieve the sum rate. To see this, note that we can find a $d_{k}^{\prime}$ such that $0<$ $d_{k}^{\prime} \leq d_{k}$ and

$$
\frac{k\left[d_{k}^{\prime-1}-\sigma_{x}^{-2}\right]^{-1}-L\left[d_{0}^{-1}-\sigma_{x}^{-2}\right]^{-1}}{L-k}=\sigma_{x}^{2} .
$$

We can show that the distortion $\left(d_{k}^{\prime}, d_{L}\right)$ with $0<d_{k}^{\prime} \leq d_{k}$ can be achieved by choosing $a=\sigma_{x}^{2}$ and $\sigma^{2}=(L-1) \sigma_{x}^{2}+L\left[d_{L}^{-1}-\sigma_{x}^{-2}\right]^{-1}$ for $\mathbf{K}_{w}$. From (10), the achievable sum rate is

$$
R=\frac{1}{2} \log \frac{\sigma_{x}^{2}}{d_{L}}
$$

Therefore, we conclude that in this case the point-to-point rate-distortion bound for the second level receiver is achievable.

$$
\text { Case 3: } \frac{k\left[d_{k}^{-1}-\sigma_{x}^{-2}\right]^{-1}-L\left[d_{L}^{-1}-\sigma_{x}^{-2}\right]^{-1}}{L-k} \leq 0 .
$$

In this case as well, the condition $0<a<\sigma_{x}^{2}$ does not hold. However, we can find a $d_{L}^{\prime}$ such that $0<d_{L}^{\prime} \leq d_{L}$ and

$$
\frac{k\left[d_{k}^{-1}-\sigma_{x}^{-2}\right]^{-1}-L\left[d_{L}^{\prime-1}-\sigma_{x}^{-2}\right]^{-1}}{L-k}=0 .
$$


We can show that the distortion $\left(d_{k}, d_{L}^{\prime}\right)$ with $0<d_{L}^{\prime} \leq d_{L}$ can be achieved by choosing $\sigma^{2}=$ $k\left[d_{k}^{-1}-\sigma_{x}^{-2}\right]^{-1}$ and $a=0$ for $\mathbf{K}_{w}$. From (10), the achievable sum rate is

$$
R=\frac{L}{2 k} \log \frac{\sigma_{x}^{2}}{d_{k}}
$$

We conclude that in this case the point-to-point rate-distortion bound for the first level receiver is achievable.

In summary, we have shown that the analog-digital separation architecture from Figure 2 achieves the lower bound to the sum rate for scalar Gaussian source.

\subsection{Vector Gaussian Source}

Now we are ready to consider the general vector situation: the memoryless source has marginal distribution $\mathcal{N}\left(0, \mathbf{K}_{x}\right)$. Let the distortion constraints be $\left(\mathbf{D}_{k}, \mathbf{D}_{L}\right)$, with

$$
\mathbf{0} \preceq \mathbf{D}_{L} \preceq \mathbf{D}_{k} \preceq \mathbf{K}_{x}
$$

Consider the analog-digital separation architecture from Figure 2 with the choice of the parameter (covariance matrix of $\left.\left(\mathbf{w}_{1}, \ldots, \mathbf{w}_{L}\right)\right)$ to be

$$
\mathbf{K}_{w}=\left(\begin{array}{ccccc}
\Lambda & -\mathbf{A} & -\mathbf{A} & \ldots & -\mathbf{A} \\
-\mathbf{A} & \boldsymbol{\Lambda} & -\mathbf{A} & \ldots & -\mathbf{A} \\
\ldots & \ldots & \ldots & \ldots & \ldots \\
-\mathbf{A} & \ldots & -\mathbf{A} & \Lambda & -\mathbf{A} \\
-\mathbf{A} & \ldots & -\mathbf{A} & -\mathbf{A} & \Lambda
\end{array}\right)
$$

We next show that with an appropriately chosen $\mathbf{K}_{w}$ this architecture achieves the lower bound (24) to the sum rate. Our first step is to characterize the conditions for the distortions are met with equality, i.e., (35) is true. From (36) and (37), we see that

$$
\begin{aligned}
& {\left[\left(\mathbf{D}_{k}^{-1}-\mathbf{K}_{x}^{-1}\right)^{-1}+\mathbf{A}\right]^{-1}=k[\boldsymbol{\Lambda}+\mathbf{A}]^{-1},} \\
& {\left[\left(\mathbf{D}_{0}^{-1}-\mathbf{K}_{x}^{-1}\right)^{-1}+\mathbf{A}\right]^{-1}=L[\boldsymbol{\Lambda}+\mathbf{A}]^{-1} .}
\end{aligned}
$$

We can solve for $\Lambda$ and $\mathbf{A}$ in the covariance matrix $\mathbf{K}_{w}$ using this constraint to get

$$
\begin{aligned}
\mathbf{\Lambda} & =\frac{(L k-k)\left[\mathbf{D}_{k}^{-1}-\mathbf{K}_{x}^{-1}\right]^{-1}-(L k-L)\left[\mathbf{D}_{L}^{-1}-\mathbf{K}_{x}^{-1}\right]^{-1}}{L-k}, \\
\mathbf{A} & =\frac{k\left[\mathbf{D}_{k}^{-1}-\mathbf{K}_{x}^{-1}\right]^{-1}-L\left[\mathbf{D}_{L}^{-1}-\mathbf{K}_{x}^{-1}\right]^{-1}}{L-k} .
\end{aligned}
$$


If the solution satisfies

$$
\mathbf{0} \prec \mathbf{A} \prec \mathbf{K}_{x},
$$

then we can conclude, from Theorem 3, the optimality of the corresponding analog-digital separation architecture. To complete the proof of Theorem 4 , we need to address the situation when for arbitrary given distortion constraints, (40) may not have a solution $\mathbf{A}^{*}$ that satisfies (49). In the following, we show that in this case there exists a choice of the parameter $\mathbf{K}_{w}$ such that corresponding analog-digital separation architecture achieves the sum rate lower bound, and resulting in distortions $\left(\mathbf{D}_{k}^{*}, \mathbf{D}_{L}^{*}\right)$ such that $\mathbf{D}_{k}^{*} \preccurlyeq \mathbf{D}_{k}$ and $\mathbf{D}_{L}^{*} \preccurlyeq \mathbf{D}_{L}$

Proof of Theorem 4. The proof involves technique similar to those used in the proof of [11, Theorem 3]. Here we provide a sketch of the proof focusing on giving the essence of the idea of enhancing distortion constraints. Some of the detailed steps, particularly those involving perturbations and taking limits, are omitted by appropriately referring the reader to [11].

First, note that by a linear transformation at the encoders and the decoders, we have the following result on rate region for multiple description with individual and central receivers.

\section{Proposition 2.}

$$
R_{*}\left(\mathbf{K}_{x}, \mathbf{D}_{k}, \mathbf{D}_{L}\right)=R_{*}\left(\mathbf{I}, \mathbf{K}_{x}^{-\frac{1}{2}} \mathbf{D}_{k} \mathbf{K}_{x}^{-\frac{1}{2}}, \mathbf{K}_{x}^{-\frac{1}{2}} \mathbf{D}_{L} \mathbf{K}_{x}^{-\frac{1}{2}}\right) .
$$

Thus, throughout this proof we will suppose, for notation simplicity, that $\mathbf{K}_{x}=\mathbf{I}$.

With this relabelling, the equation (40) is now

$$
\frac{1}{k}\left[\boldsymbol{\Lambda}_{k}+\mathbf{A}\right]^{-1}=\frac{1}{L}\left[\boldsymbol{\Lambda}_{L}+\mathbf{A}\right]^{-1},
$$

where

$$
\begin{aligned}
& \boldsymbol{\Lambda}_{k}=\left(\mathbf{D}_{k}^{-1}-\mathbf{I}\right)^{-1} \\
& \boldsymbol{\Lambda}_{L}=\left(\mathbf{D}_{L}^{-1}-\mathbf{I}\right)^{-1}
\end{aligned}
$$

Define

$$
\begin{aligned}
& f(\mathbf{A}) \stackrel{\text { def }}{=} \frac{1}{L}\left[\boldsymbol{\Lambda}_{L}+\mathbf{A}\right]^{-1}-\frac{1}{k}\left[\boldsymbol{\Lambda}_{k}+\mathbf{A}\right]^{-1} \\
& F(\mathbf{A}) \stackrel{\text { def }}{=} \frac{1}{L} \log \left|\boldsymbol{\Lambda}_{L}+\mathbf{A}\right|-\frac{1}{k} \log \left|\boldsymbol{\Lambda}_{k}+\mathbf{A}\right|
\end{aligned}
$$

Note that

$$
\frac{d F(\mathbf{A})}{d \mathbf{A}}=f(\mathbf{A})
$$

Consider the following optimization problem:

$$
\max _{\mathbf{0} \preccurlyeq \mathbf{A} \preccurlyeq \mathbf{I}} F(\mathbf{A}) .
$$


Since $F(\mathbf{A})$ is a continuous map and $\mathbf{0} \preccurlyeq \mathbf{A} \preccurlyeq \mathbf{I}$ is a compact set, there exists an optimal solution $\mathbf{A}^{*}$ to (51) where $\mathbf{A}^{*}$ satisfies the Karush-Kuhn-Tucker (KKT) conditions [12, Section 5.5.3]: there exist $\Gamma_{1} \succcurlyeq 0$ and $\Gamma_{2} \succcurlyeq 0$ such that

$$
\begin{aligned}
f\left(\mathbf{A}^{*}\right)+\frac{1}{L} \boldsymbol{\Gamma}_{1}-\frac{1}{k} \boldsymbol{\Gamma}_{2} & =\mathbf{0} \\
\boldsymbol{\Gamma}_{1} \mathbf{A}^{*} & =\mathbf{0} \\
\boldsymbol{\Gamma}_{2}\left(\mathbf{A}^{*}-\mathbf{I}\right) & =\mathbf{0} .
\end{aligned}
$$

Now $\mathbf{A}^{*}$ falls into the following four cases.

Case 1: $0 \prec \mathbf{A}^{*} \prec \mathbf{I}$. Alternatively, 0 and 1 are not eigenvalues of $\mathbf{A}^{*}$. In this case, $\boldsymbol{\Gamma}_{1}=\mathbf{0}$ and $\Gamma_{2}=0$; thus the KKT conditions in (57) reduce to

$$
f\left(\mathbf{A}^{*}\right)=\mathbf{0} .
$$

From Theorem 3, the analog-digital separation architecture with covariance matrix for $\mathbf{w}_{1}, \ldots, \mathbf{w}_{L}$ taking form (46) achieves the lower bound to the sum rate, and the $\Lambda$ and $\mathbf{A}$ in (46) are given in (48).

Case 2: $\mathbf{0} \preccurlyeq \mathbf{A}^{*} \prec \mathbf{I}$. Alternatively, some eigenvalues of $\mathbf{A}^{*}$ are 0 , but no eigenvalues of $\mathbf{A}^{*}$ are 1. Thus $\Gamma_{2}=\mathbf{0}$ and the KKT conditions in (57) reduce to

$$
\frac{1}{L}\left[\boldsymbol{\Lambda}_{L}+\mathbf{A}\right]^{-1}-\frac{1}{k}\left[\boldsymbol{\Lambda}_{k}+\mathbf{A}\right]^{-1}+\frac{1}{L} \boldsymbol{\Gamma}_{1}=\mathbf{0},
$$

for some $\Gamma_{1} \succcurlyeq 0$ satisfying $\Gamma_{1} \mathbf{A}^{*}=0$. The key idea now is to see that the distortion constraint on the second level receiver is too loose and we can in fact achieve a lower distortion (in the sense of positive semidefinite ordering) for the same sum rate. We first identify this lower distortion: defining

$$
\Lambda_{L}^{*}=\left(\Lambda_{L}^{-1}+\Gamma_{1}\right)^{-1}
$$

consider the smaller distortion matrix on the central receiver

$$
\mathbf{D}_{L}^{*}=\left(\boldsymbol{\Lambda}_{L}^{*-1}+\mathbf{I}\right)^{-1}=\left(\mathbf{I}+\boldsymbol{\Lambda}_{L}^{-1}+\boldsymbol{\Gamma}_{1}\right)^{-1}=\left(\mathbf{D}_{L}^{-1}+\boldsymbol{\Gamma}_{1}\right)^{-1} \prec \mathbf{D}_{L} .
$$

From [11, Lemma 6] we know that this new distortion matrix on the second level receiver satisfies the following two key properties.

\section{Lemma 4.}

$$
\begin{aligned}
\left(\boldsymbol{\Gamma}_{L}+\mathbf{A}^{*}\right)^{-1}+\boldsymbol{\Gamma}_{1} & =\left(\boldsymbol{\Gamma}_{L}^{*}+\mathbf{A}^{*}\right)^{-1} \\
\frac{\left|\mathbf{D}_{L}+\mathbf{K}_{z}\right|}{\left|\mathbf{D}_{L}\right|} & =\frac{\left|\mathbf{D}_{L}^{*}+\mathbf{K}_{z}\right|}{\left|\mathbf{D}_{L}^{*}\right|}
\end{aligned}
$$

Proof. The proof in available in Appendix B; it closely follows Lemma 5 in [11] and we provide it here for completeness. 
Comparing (60) with (63), we have

$$
\frac{1}{L}\left[\boldsymbol{\Lambda}_{L}^{*}+\mathbf{A}^{*}\right]^{-1}=\frac{1}{k}\left[\boldsymbol{\Lambda}_{k}+\mathbf{A}^{*}\right]^{-1} .
$$

We consider the enhanced problem with modified distortion constraint $\left(\mathbf{D}_{k}, \mathbf{D}_{L}^{*}\right)$. For the enhanced problem, we have a solution $\mathbf{A}^{*}$ to (40), but the corresponding $\mathbf{K}_{z}=\left(\mathbf{I}-\mathbf{A}^{*}\right)^{-1}-\mathbf{I}$ is singular. This technical difficulty can be addressed by first perturbing $\mathbf{A}^{*}$ to $\mathbf{A}^{*}+\epsilon \mathbf{I}$, then letting $\epsilon \rightarrow 0$, as was done in [11] and we can formally use $\mathbf{K}_{z}$ as if it were not singular. By Theorem 3, we conclude that the analog-digital separation architecture is optimal to achieving the lower bound to the sum rate of the enhanced problem. From (64) we can see that the enhanced problem and the original problem have the same lower bound to the sum rate (41). Hence the analog-digital separation architecture also achieves lower bound to the sum rate of the original problem, and the resulting distortion $\left(\mathbf{D}_{k}, \mathbf{D}_{L}^{*}\right)$ satisfies $\mathbf{D}_{L}^{*} \preccurlyeq \mathbf{D}_{L}$.

Case 3: $\mathbf{0} \prec \mathbf{A}^{*} \preccurlyeq \mathbf{I}$. Alternatively, some eigenvalues of $\mathbf{A}^{*}$ are 1 , but no eigenvalues of $\mathbf{A}^{*}$ are 0 . In this case, $\Gamma_{1}=0$ and the KKT conditions in (57) reduce to

$$
\frac{1}{L}\left[\boldsymbol{\Lambda}_{L}+\mathbf{A}^{*}\right]^{-1}-\frac{1}{k}\left[\boldsymbol{\Lambda}_{k}+\mathbf{A}^{*}\right]^{-1}-\frac{1}{k} \boldsymbol{\Gamma}_{2}=0,
$$

for some $\boldsymbol{\Gamma}_{2} \succcurlyeq 0$ satisfying $\boldsymbol{\Gamma}_{2}\left(\mathbf{A}^{*}-\mathbf{I}\right)=\mathbf{0}$. Defining

$$
\boldsymbol{\Lambda}_{k}^{*}=\left[\left(\boldsymbol{\Lambda}_{k}+\mathbf{I}\right)^{-1}+\boldsymbol{\Gamma}_{2}\right]^{-1}-\mathbf{I}
$$

we have, as in (63), that

$$
\left(\boldsymbol{\Lambda}_{k}+\mathbf{A}^{*}\right)^{-1}+\boldsymbol{\Gamma}_{2}=\left(\boldsymbol{\Lambda}_{k}^{*}+\mathbf{A}^{*}\right)^{-1} .
$$

Now, from (68), we have

$$
\frac{1}{L}\left[\boldsymbol{\Lambda}_{L}+\mathbf{A}^{*}\right]^{-1}=\frac{1}{k}\left[\boldsymbol{\Lambda}_{k}^{*}+\mathbf{A}^{*}\right]^{-1} .
$$

Define $\mathbf{D}_{k}^{*}=\left[\boldsymbol{\Lambda}_{k}^{*-1}+\mathbf{I}\right]^{-1}$, we have

$$
\begin{aligned}
\mathbf{D}_{k}^{*} & =\left[\boldsymbol{\Lambda}_{k}^{*-1}+\mathbf{I}\right]^{-1} \\
& =\left[\left(\left(\left(\boldsymbol{\Lambda}_{k}+\mathbf{I}\right)^{-1}+\boldsymbol{\Gamma}_{2}\right)^{-1}-\mathbf{I}\right)^{-1}+\mathbf{I}\right]^{-1} \\
& =\left[\mathbf{I}-\left(\boldsymbol{\Lambda}_{k}+\mathbf{I}\right)^{-1}-\boldsymbol{\Gamma}_{2}\right] \\
& =\left[\mathbf{I}+\boldsymbol{\Lambda}_{k}\right]^{-1}-\boldsymbol{\Gamma}_{2} \\
& =\mathbf{D}_{k}-\boldsymbol{\Gamma}_{2} .
\end{aligned}
$$

We now consider the enhanced problem with modified distortion constraint $\left(\mathbf{D}_{k}^{*}, \mathbf{D}_{L}\right)$. For the enhanced problem, we have a solution $\mathbf{A}^{*}$ to (40), but $\left(\mathbf{I}-\mathbf{A}^{*}\right)$ is singular. This technical difficulty can also be addressed by first perturbing $\mathbf{A}^{*}$ to $\mathbf{A}^{*}-\epsilon \mathbf{I}$, then letting $\epsilon \rightarrow 0$, as was done in [11], and we can formally treat $\mathbf{K}_{z}=\lambda \mathbf{I}, \lambda \rightarrow \infty$. By Theorem 3, we conclude that the analog-digital 
separation architecture is optimal in terms of achieving the lower bound to the sum rate of the enhanced problem, which is

$$
\begin{aligned}
& \sup _{\mathbf{K}_{z}=\lambda \mathbf{I}, \lambda \rightarrow \infty}\left\{\frac{L}{k\left(\begin{array}{l}
L \\
k
\end{array}\right)}\left[\frac{1}{2} \log \frac{\left|\mathbf{K}_{x}+\mathbf{K}_{z}\right|}{\left|\mathbf{D}_{k}+\mathbf{K}_{z}\right|}\right]+\frac{1}{2} \log \frac{\left|\mathbf{K}_{x}\right|}{\left|\mathbf{K}_{x}+\mathbf{K}_{z}\right|}+\frac{1}{2} \log \frac{\left|\mathbf{D}_{L}+\mathbf{K}_{z}\right|}{\left|\mathbf{D}_{L}\right|}\right\} \\
= & \frac{1}{2} \log \frac{\left|\mathbf{K}_{x}\right|}{\left|\mathbf{D}_{L}\right|} .
\end{aligned}
$$

Note this is the point-to-point rate-distortion function for the second layer receiver with distortion $\mathbf{D}_{L}$, which is also a lower bound to the sum rate to the original problem. Hence analog-digital separation architecture also achieves a lower bound to the sum rate to the original problem, and the resulting distortion $\left(\mathbf{D}_{k}^{*}, \mathbf{D}_{L}\right)$ satisfies $\mathbf{D}_{k}^{*} \preccurlyeq \mathbf{D}_{k}$.

Case 4: $0 \preccurlyeq \mathbf{A}^{*} \preccurlyeq \mathbf{I}$. i.e., both 0 and 1 are eigenvalues of $\mathbf{A}^{*}$. In this case, the KKT conditions are: there exist $\boldsymbol{\Gamma}_{1} \succcurlyeq 0$ and $\boldsymbol{\Gamma}_{2} \succcurlyeq 0$ such that equations (57), (58) and (59) hold. We can combine equations (63) and (68) to get

$$
\frac{1}{L}\left(\boldsymbol{\Lambda}_{L}^{*}+\mathbf{A}^{*}\right)^{-1}=\frac{1}{k}\left(\boldsymbol{\Lambda}_{k}+\mathbf{A}^{*}\right)^{-1}
$$

where

$$
\begin{aligned}
\boldsymbol{\Lambda}_{L}^{*} & =\left(\boldsymbol{\Lambda}_{L}^{-1}+\boldsymbol{\Gamma}_{1}\right)^{-1}, \\
\boldsymbol{\Lambda}_{k}^{*} & =\left[\left(\boldsymbol{\Lambda}_{k}+\mathbf{I}\right)^{-1}+\boldsymbol{\Gamma}_{2}\right]^{-1}-\mathbf{I} .
\end{aligned}
$$

We now consider the enhanced problem with modified distortion constraint $\left(\mathbf{D}_{k}^{*}, \mathbf{D}_{L}^{*}\right)$, where

$$
\begin{aligned}
\mathbf{D}_{k}^{*} & =\mathbf{D}_{k}-\boldsymbol{\Gamma}_{2}, \\
\mathbf{D}_{L}^{*} & =\left(\mathbf{D}_{L}^{-1}+\boldsymbol{\Gamma}_{1}\right)^{-1} .
\end{aligned}
$$

For the enhanced problem, we have a solution $\mathbf{A}^{*}$ to (40), and by properly perturbing $\mathbf{A}^{*}$ and taking limits, we can show that the analog-digital separation architecture is optimal to achieve the point-to-point rate-distortion function for the second layer receiver with distortion $\mathbf{D}_{L}$. Hence analog-digital separation architecture also achieves a lower bound to the sum rate to the original problem, and the resulting distortion $\left(\mathbf{D}_{k}^{*}, \mathbf{D}_{L}^{*}\right)$ satisfies $\mathbf{D}_{k}^{*} \preccurlyeq \mathbf{D}_{k}, \mathbf{D}_{L}^{*} \preccurlyeq \mathbf{D}_{L}$.

To summarize, we see that the analog-digital separation architecture achieves the limiting sum rate. The limiting sum rate is the solution to an optimization problem. For some specific distortion constraints, the sum rate can be characterized as the solution to a matrix equation (Case 1). 


\section{Example: $k=L-1$}

In this section we provide another example where the lower bound to sum rate (24) can be achieved by the analog-digital separation architecture. We consider the case where the first level decoder can receive any combination of $L-1$ descriptions. We first assume that (10) is the achievable sum rate. In this case, there are $L+1$ distortion constraints. We consider the analog-digital separation architecture with the covariance matrix $\mathbf{K}_{w}$ for $\left(\mathbf{w}_{1}, \ldots, \mathbf{w}_{L}\right)$ taking the form of (32). We need to solve $\mathbf{K}_{w}$ in (32) from the conditions that all distortion constraints are met with equality. When $k=L-1$, these conditions are

$$
\begin{aligned}
& {\left[\left(\mathbf{D}_{S}^{-1}-\mathbf{K}_{x}^{-1}\right)^{-1}+\mathbf{A}\right]^{-1}=\sum_{l \in S}\left[\mathbf{K}_{w_{l}}+\mathbf{A}\right]^{-1}, \quad \forall S \in\{1,2, \cdots, L\},|S|=L-1} \\
& {\left[\left(\mathbf{D}_{L}^{-1}-\mathbf{K}_{x}^{-1}\right)^{-1}+\mathbf{A}\right]^{-1}=\sum_{l=1}^{L}\left[\mathbf{K}_{w_{l}}+\mathbf{A}\right]^{-1} .}
\end{aligned}
$$

Define

$$
\begin{aligned}
& \boldsymbol{\Lambda}_{S}=\left(\mathbf{D}_{S}^{-1}-\mathbf{K}_{x}^{-1}\right)^{-1}, \quad \forall S \in\{1,2, \cdots, L\},|S|=L-1, \\
& \boldsymbol{\Lambda}_{L}=\left(\mathbf{D}_{L}^{-1}-\mathbf{K}_{x}^{-1}\right)^{-1} .
\end{aligned}
$$

We have the following equation for $\mathbf{A}$.

$$
\frac{1}{k} \sum_{S,|S|=L-1}\left[\boldsymbol{\Lambda}_{S}+\mathbf{A}\right]^{-1}=\left[\boldsymbol{\Lambda}_{L}+\mathbf{A}\right]^{-1}
$$

We can also solve $\mathbf{K}_{w_{l}}$ from the following equation

$$
\left[\mathbf{K}_{w_{l}}+\mathbf{A}\right]^{-1}=\left[\boldsymbol{\Lambda}_{L}+\mathbf{A}\right]^{-1}-\left[\boldsymbol{\Lambda}_{S}+\mathbf{A}\right]^{-1},
$$

where $S=\{1, \ldots, l-1, l+1, \ldots, L\}$. If the solution of (77) satisfies $\mathbf{0} \prec \mathbf{A} \prec \mathbf{K}_{x}$, then from

Theorem 3 we know that the analog-digital separation architecture achieves the optimal sum rate. When the solution does not satisfy $\mathbf{0} \prec \mathbf{A} \prec \mathbf{K}_{x}$, define

$$
\begin{aligned}
& f(\mathbf{A}) \stackrel{\text { def }}{=}\left[\boldsymbol{\Lambda}_{L}+\mathbf{A}\right]^{-1}-\frac{1}{k} \sum_{S,|S|=L-1}\left[\boldsymbol{\Lambda}_{S}+\mathbf{A}\right]^{-1} \\
& F(\mathbf{A}) \stackrel{\text { def }}{=} \log \left|\boldsymbol{\Lambda}_{L}+\mathbf{A}\right|-\frac{1}{k} \sum_{S,|S|=L-1} \log \left|\boldsymbol{\Lambda}_{k}+\mathbf{A}\right| .
\end{aligned}
$$

Note that

$$
\frac{d F(\mathbf{A})}{d \mathbf{A}}=f(\mathbf{A})
$$

Consider the following optimization problem:

$$
\max _{\mathbf{0} \preccurlyeq \mathbf{A} \preccurlyeq \mathbf{K}_{x}} F(\mathbf{A}) .
$$


We can connect $\mathbf{A}^{*}$, the solution to (77), to the optimization problem described above. By using techniques similar to those in the proof of Theorem 4, we can show that there exists a analog-digital separation architecture that achieves the sum rate lower bound, and results in distortions $\left(\mathbf{D}_{S}^{*}, \mathbf{D}_{L}^{*}\right)$ such that $\mathbf{D}_{S}^{*} \preccurlyeq \mathbf{D}_{S}$ for $\forall S \in\{1,2, \cdots, L\},|S|=L-1$, and $\mathbf{D}_{L}^{*} \preccurlyeq \mathbf{D}_{L}$. We omit the complete details due to the close similarity to the proof of Theorem 4.

From the above discussion, we can see that if (10) is achievable, then the analog-digital separation architecture is optimal. From Lemma 3 we know that when $k=2$ and $L=3,(10)$ is always achievable. Thus we have the following result.

Theorem 5. For $k=2$ and $L=3$, given distortion constraints $\left(\mathbf{D}_{S}, \mathbf{D}_{L}\right)$, the optimal sum rate is

$$
\frac{1}{2} \sum_{|S|=2}\left[\frac{1}{2} \log \frac{\left|\mathbf{K}_{x}+\mathbf{K}_{z}\right|}{\left|\mathbf{D}_{S}+\mathbf{K}_{z}\right|}\right]+\frac{1}{2} \log \frac{\left|\mathbf{K}_{x}\right|}{\left|\mathbf{K}_{x}+\mathbf{K}_{z}\right|}+\frac{1}{2} \log \frac{\left|\mathbf{D}_{L}+\mathbf{K}_{z}\right|}{\left|\mathbf{D}_{L}\right|} .
$$

\section{Discussion}

\subsection{Robustness of Gaussian Multiple Descriptions}

In this section, we show that the distortion achieved through describing any memoryless vector source using the analog-digital separation architecture is no worse (in the sense of positive semidefinite ordering) than when the source itself were Gaussian (among all sources with the same second order statistics). This is a demonstration of the robustness of the separation architecture. Further, this result implies that the Gaussian memoryless vector source is the hardest to multiply describe among all memoryless vector sources with the same covariance matrix.

Proposition 3. Consider a memoryless vector source $\mathbf{x}^{n}$ with marginal covariance matrix $\mathbf{K}_{x}$ being multiply described by the analog-digital separation architecture using the parameter $\mathbf{K}_{w}$ (cf. Section 3). As usual the decoders reconstruct via the MMSE estimation. Then, the achieved distortions $\left(\mathbf{D}_{S}, \mathbf{D}_{L}\right)$ satisfy

$$
\begin{aligned}
& \mathbf{D}_{S} \preceq \mathbf{D}_{S}^{G} \quad \forall S \subset\{1, \ldots, L\},|S|=k, \\
& \mathbf{D}_{L} \preceq \mathbf{D}_{L}^{G}
\end{aligned}
$$

where $\left(\mathbf{D}_{S}^{G}, \mathbf{D}_{L}^{G}\right)$ is the distortion by the same architecture achieved when $\mathbf{x}^{n}$ is a memoryless vector Gaussian source.

Proof. Using the analog-digital separation architecture described in Section 3, let

$$
\mathbf{u}_{l}=\mathbf{x}+\mathbf{w}_{l}, \quad l=1, \ldots, L .
$$


We first consider the following reconstruction

$$
\begin{aligned}
& \hat{\mathbf{x}}_{S}=\sum_{l \in S} \alpha_{l}^{S} \mathbf{u}_{l}, \quad \forall S \subset 1, \ldots, L,|S|=k, \\
& \hat{\mathbf{x}}_{L}=\sum_{l=1}^{L} \beta_{l} \mathbf{u}_{l},
\end{aligned}
$$

where $\alpha$ 's and $\beta$ 's are chosen so that $\sum_{l \in S} \alpha_{l}^{S} \mathbf{u}_{l}$ is the linear MMSE estimation of $\mathbf{x}$ given $\mathbf{u}_{l}$ for

$\forall S \subset 1, \ldots, L,|S|=k$, and $\sum_{i=1}^{L} \beta_{l} \mathbf{u}_{l}$ is the linear MMSE estimation of $\mathbf{x}$ given $\mathbf{u}_{1}, \cdots, \mathbf{u}_{L}$. We can see that all the $\alpha$ 's and $\beta$ 's do not change with the source distribution for fixed analog-digital separation architecture. The distortions achieved are

$$
\begin{aligned}
& \left.\mathbf{D}_{S}=\mathbb{E}\left[\left(\mathbf{x}-\hat{\mathbf{x}}_{S}\right)^{t}\left(\mathbf{x}-\hat{\mathbf{x}}_{S}\right]\right)\right], \quad \forall S \subset 1, \ldots, L,|S|=k, \\
& \mathbf{D}_{L}=\mathbb{E}\left[\left(\mathbf{x}-\hat{\mathbf{x}}_{L}\right)^{t}\left(\mathbf{x}-\hat{\mathbf{x}}_{L}\right)\right] .
\end{aligned}
$$

Note that if $\mathbf{x}^{n}$ is i.i.d vector Gaussian, then linear MMSE is the MMSE estimation, and if $\mathbf{x}^{n}$ is not Gaussian, then MMSE estimation leads to smaller distortion then the linear MMSE estimation, we have (84).

\subsection{Non-Gaussian Sources}

Using this result we can now conclude that the Gaussian statistics poses the hardest trouble for multiple description with quadratic distortion constraints. To see this, observe that the sum rate of multiple description using a fixed analog-digital separation architecture satisfies

$$
\sum_{l=1}^{L} R_{l} \geq \frac{L}{k\left(\begin{array}{l}
L \\
k
\end{array}\right)}\left[\sum_{|S|=k} h\left(\mathbf{u}_{S}\right)\right]-h\left(\mathbf{u}_{1}, \ldots, \mathbf{u}_{L} \mid \mathbf{x}\right)
$$

Because $h\left(\mathbf{u}_{S}\right)$ is maximized when $\mathbf{x}$ is Gaussian, we have

$$
\frac{L}{k\left(\begin{array}{l}
L \\
k
\end{array}\right)}\left[\sum_{|S|=k} h\left(\mathbf{u}_{S}\right)\right]-h\left(\mathbf{u}_{1}, \ldots, \mathbf{u}_{L} \mid \mathbf{x}\right) \leq \frac{L}{k\left(\begin{array}{l}
L \\
k
\end{array}\right)} \frac{1}{2} \log \left[\prod_{|S|=k}\left|\mathbf{K}_{u_{S}}\right|\right]-\frac{1}{2} \log \left|\mathbf{K}_{w}\right| .
$$

Thus we have the following upper bound on the achievable sum rate for arbitrary vector source $\mathrm{x}$.

Corollary 1. Consider a memoryless vector source $\mathbf{x}^{n}$ with given marginal covariance matrix $\mathbf{K}_{x}$. When (10) is achievable, the optimal sum rate in compressing this $\mathrm{x}^{n}$ in the multiple description 
problems for given distortion constraint $\left(\mathbf{D}_{S}, \mathbf{D}_{L}\right)$ is upper bounded by

$$
\min _{\mathbf{K}_{w}} \frac{L}{k\left(\begin{array}{l}
L \\
k
\end{array}\right)} \frac{1}{2} \log \left[\prod_{|S|=k}\left|\mathbf{K}_{u_{S}}\right|\right]-\frac{1}{2} \log \left|\mathbf{K}_{w}\right|
$$

where the minimization is over all $\mathbf{K}_{w}$ so that the reconstructing sequences satisfy distortion constraints.

We can also derive a lower bound on sum rate. Define normalized entropy power for $N$ dimension random vector $\mathrm{x}$ as

$$
P_{\mathbf{x}}=\left(\frac{e^{\frac{2}{N}} h(\mathbf{x})}{2 \pi e}\right)^{N}
$$

we have the following result.

Proposition 4. Consider a memoryless vector source $\mathrm{x}^{n}$ with given marginal covariance matrix $\mathbf{K}_{x}$. The optimal sum rate in the multiple description problems for given distortion constraint $\left(\mathbf{D}_{S}, \mathbf{D}_{L}\right)$ is lower bounded by

$$
\sup _{\mathbf{K}_{z} \succeq \mathbf{0}} \frac{L}{k\left(\begin{array}{l}
L \\
k
\end{array}\right)} \sum_{|S|=k}\left[\frac{1}{2} \log \frac{\left(P_{\mathbf{x}}^{1 / N}+\left|\mathbf{K}_{z}\right|^{1 / N}\right)^{N}}{\left|\mathbf{D}_{S}+\mathbf{K}_{z}\right|}\right]+\frac{1}{2} \log \frac{P_{\mathbf{x}}}{\left(P_{\mathbf{x}}^{1 / N}+\left|\mathbf{K}_{z}\right|^{1 / N}\right)^{N}}+\frac{1}{2} \log \frac{\left|\mathbf{D}_{L}+\mathbf{K}_{z}\right|}{\left|\mathbf{D}_{L}\right|} .
$$

Proof. We can see that every step in the proof of Theorem 1 still holds for arbitrarily distributed i.i.d vector source $\mathrm{x}^{n}$ except for equation (26), which becomes

$$
\begin{aligned}
h\left(\mathbf{x}^{n}\right) & =\frac{1}{2} \log (2 \pi e)^{N n} P_{\mathbf{x}}^{n}, \\
h\left(\mathbf{y}^{n}\right) & =\frac{1}{2} \log (2 \pi e)^{N n} P_{\mathbf{y}}^{n} \geq \frac{1}{2} \log (2 \pi e)^{N n}\left(P_{\mathbf{x}}^{1 / N}+\left|\mathbf{K}_{z}\right|^{1 / N}\right)^{N n} .
\end{aligned}
$$

Following the rest of the steps in proof of Theorem 1, we get the lower bound.

\subsection{Problem of Distributed Observation}

In the setting of the multiple description problem, all the encoders are located at the same place as the source $\mathbf{x}^{n}$. However, in practice the observations about the same may be distributed. Consider the following multiple description problem with distributed observation (see Figure 3): Given an information source $\mathrm{x}^{n}$, there are $L$ distributed sensors, each of them observes this information 


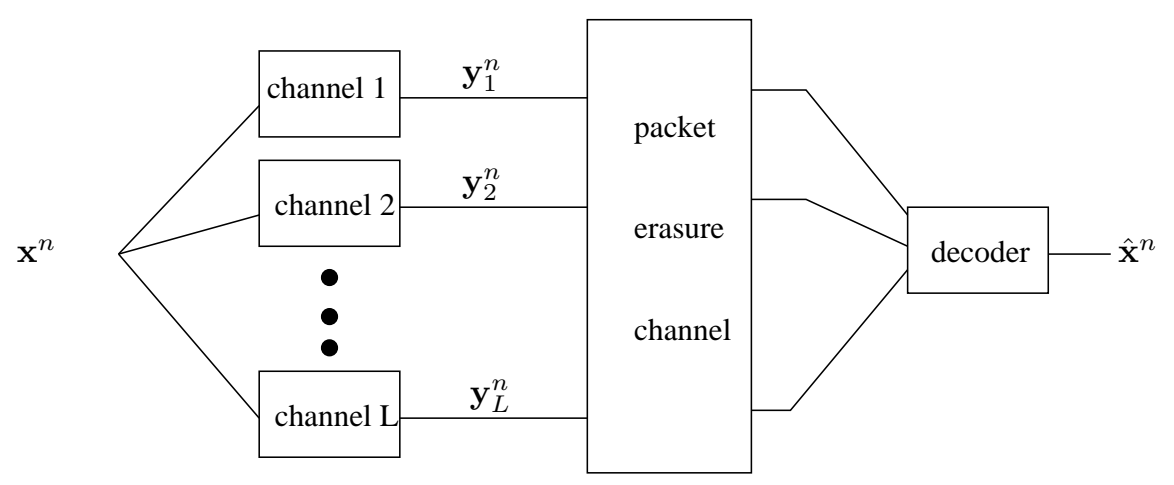

Figure 3: Multiple description problem with distributed observation

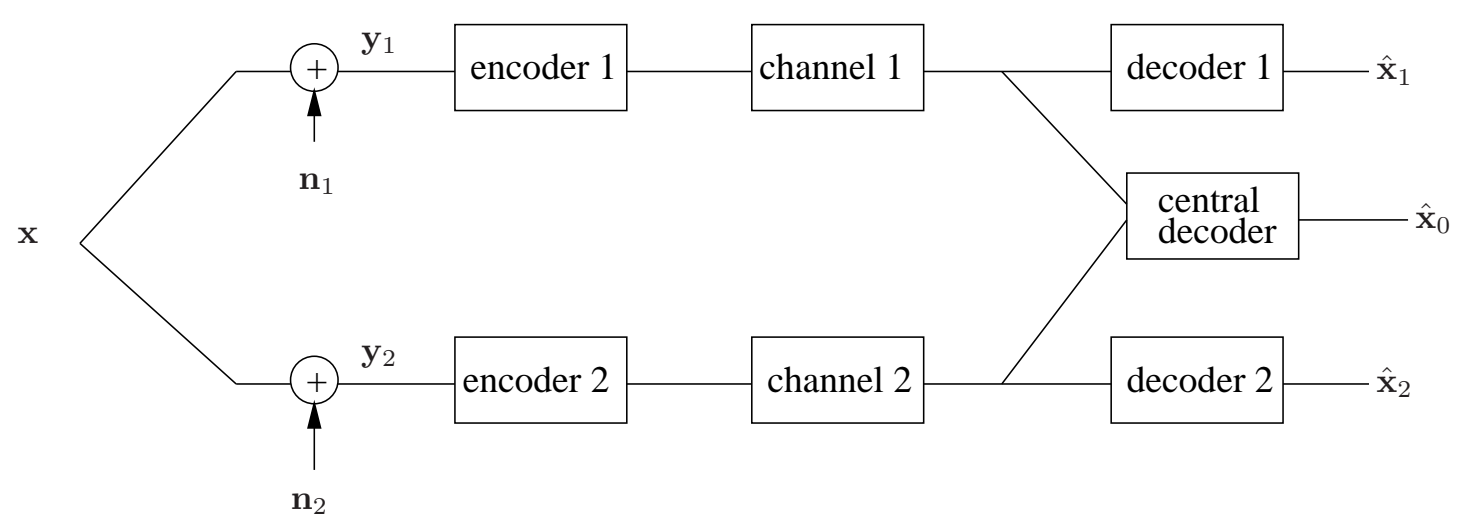

Figure 4: Robust CEO problem.

source through a communication channel. Each sensor generates a description of the observation, and the $L$ descriptions are sent through $L$ separate unreliable channels to one destination. Either (an arbitrary subset) $k$ of the descriptions are received, or all descriptions are received. The destination needs to regenerate he source $\mathrm{x}^{n}$ based on the descriptions it received, subject to some distortion constraint.

When the channels from the information source to the sensors are noisy, the first step in the analog-digital separation architecture breaks down because now the sensors do not know each other's observation and they can not perform joint descriptions any more. When $k=1$ and $L=2$, this problem is studied under the name of robust CEO problem in [13] (see Figure 4 for an example when the channels are additive noise channel). The achievable scheme given in [13] has a twolayer structure. The codebooks generated at both encoders have two parts. The first part is the vector quantization of the source sequence and it satisfies the individual distortion constraint. And the second part is a codebook for CEO problem of the central decoder, taking the first part as side information. 


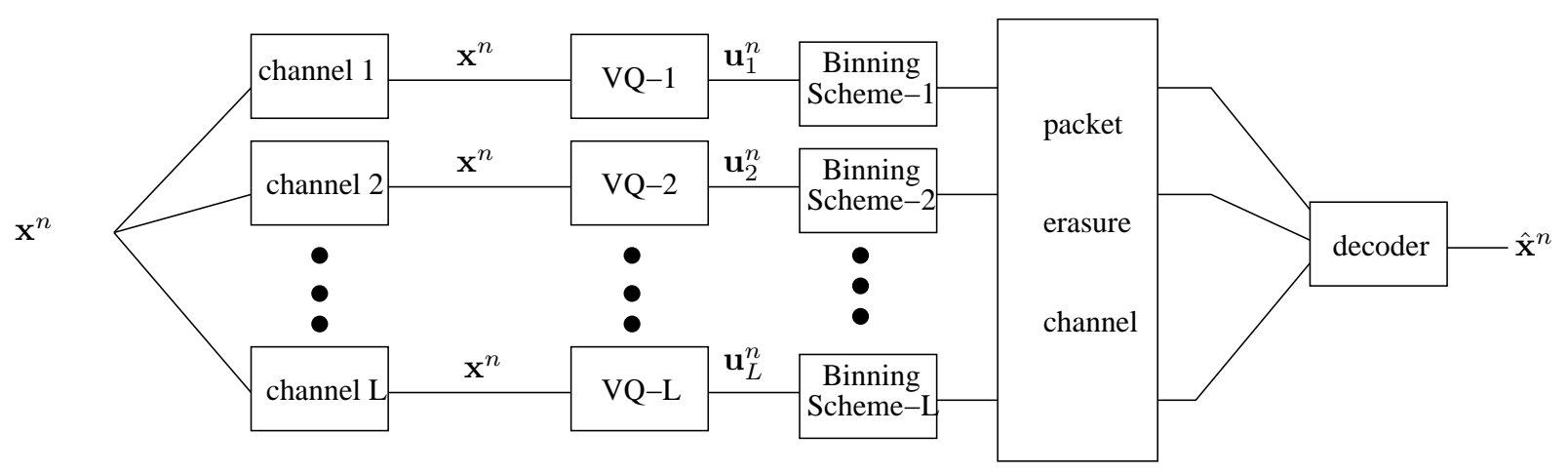

Figure 5: Analog-digital separation architecture for multiple description problem with distributed perfect observation

On the other hand, if the channels from the information source to all the sensors are perfect channel, i.e., all the sensors observe the same $\mathbf{x}^{n}$, then the natural analog-digital separation scheme described in this paper is well suited to provide multiple description for this problem (see Figure 5). The first step in the analog-digital separation scheme is not changed, the mapping from $\mathrm{x}^{n}$ to $\mathbf{u}^{n}$ 's are jointly fixed and assigned to each sensors, and in the second step a distributed binning scheme is used, which does not require the co-location of the $\mathbf{u}^{n}$ 's.

\section{Appendix}

\section{A Proof of Proposition 1}

Conditioned on $\mathbf{y}$, the collection of random variables $\mathbf{u}_{S}, \forall S \subset 1, \ldots, L$ are Gaussian and thus we have

$$
\sum_{l \in S} h\left(\mathbf{u}_{l} \mid \mathbf{y}\right)-h\left(\mathbf{u}_{S} \mid \mathbf{y}\right)=\frac{1}{2} \log \frac{\prod_{l \in S}\left|\operatorname{Cov}\left[\mathbf{u}_{l} \mid \mathbf{y}\right]\right|}{\left|\operatorname{Cov}\left[\mathbf{u}_{S} \mid \mathbf{y}\right]\right|} .
$$

From MMSE of $\mathbf{u}_{l}$ from $\mathbf{y}$ we have

$$
\operatorname{Cov}\left[\mathbf{u}_{l} \mid \mathbf{y}\right]=\mathbf{K}_{x}+\mathbf{K}_{w_{l}}-\mathbf{K}_{x}\left(\mathbf{K}_{x}+\mathbf{K}_{z}\right)^{-1} \mathbf{K}_{x}, \quad l=1, \ldots, L
$$

and

$$
\operatorname{Cov}\left(\mathbf{u}_{S} \mid \mathbf{y}\right)=\mathbf{J} \otimes \mathbf{K}_{x}+\mathbf{K}_{w_{S}}-\mathbf{J} \otimes\left(\mathbf{K}_{x}\left(\mathbf{K}_{x}+\mathbf{K}_{z}\right)^{-1} \mathbf{K}_{x}\right),
$$

where $\mathbf{J}$ is an $|S| \times|S|$ matrix of all ones and $\otimes$ is the Kronecker Product [10, Section 6.5].

By Fischer inequality (the block matrix version of Hadamard inequality, see [10, Theorem 6.10]) we know that $\prod_{l \in S}\left|\operatorname{Cov}\left[\mathbf{u}_{l} \mid \mathbf{y}\right]\right|=\left|\operatorname{Cov}\left[\mathbf{u}_{S} \mid \mathbf{y}\right]\right|$ if and only if the off-diagonal block matrices 
of $\operatorname{Cov}\left[\mathbf{u}_{S} \mid \mathbf{y}\right]$ are all zero matrices. Thus we have

$$
\sum_{l \in S} h\left(\mathbf{u}_{l} \mid \mathbf{y}\right)-h\left(\mathbf{u}_{S} \mid \mathbf{y}\right)=0
$$

if and only if

$$
\mathbf{K}_{x}-\mathbf{A}=\mathbf{K}_{x}\left(\mathbf{K}_{x}+\mathbf{K}_{z}\right)^{-1} \mathbf{K}_{x}
$$

or equivalently, if and only if

$$
\mathbf{K}_{z}=\mathbf{K}_{x}\left(\mathbf{K}_{x}-\mathbf{A}\right)^{-1} \mathbf{K}_{x}-\mathbf{K}_{x}
$$

Letting $|S|=k$, we have

$$
\sum_{l \in S} h\left(\mathbf{u}_{l} \mid \mathbf{y}\right)=h\left(\mathbf{u}_{S} \mid \mathbf{y}\right), \quad \forall S \subset\{1, \ldots, L\},|S|=k .
$$

Letting $|S|=L$, we have

$$
\sum_{l=1}^{L} h\left(\mathbf{u}_{l} \mid \mathbf{y}\right)=h\left(\mathbf{u}_{1}, \ldots, \mathbf{u}_{L} \mid \mathbf{y}\right)
$$

and it is readily seen that (34) is true.

To get a valid $\mathbf{K}_{z} \succ \mathbf{0}$, we need the additional condition $\mathbf{0} \prec \mathbf{A} \prec \mathbf{K}_{x}$.

\section{B Proof of Lemma 4}

$$
\begin{aligned}
{\left[\left(\boldsymbol{\Lambda}_{L}+\mathbf{A}^{*}\right)^{-1}+\boldsymbol{\Gamma}_{1}\right]^{-1} } & =\left[\left(\boldsymbol{\Lambda}_{L}+\mathbf{A}^{*}\right)^{-1}\left(\mathbf{I}+\left(\boldsymbol{\Lambda}_{L}+\mathbf{A}^{*}\right) \boldsymbol{\Gamma}_{1}\right)\right]^{-1} \\
& \stackrel{(a)}{=}\left(\mathbf{I}+\boldsymbol{\Lambda}_{L} \boldsymbol{\Gamma}_{1}\right)^{-1}\left(\boldsymbol{\Lambda}_{L}+\mathbf{A}^{*}\right) \\
& =\left(\mathbf{I}+\boldsymbol{\Lambda}_{L} \boldsymbol{\Gamma}_{1}\right)^{-1}\left(\boldsymbol{\Lambda}_{L}+\mathbf{A}^{*}-\left(\mathbf{I}+\boldsymbol{\Lambda}_{L} \boldsymbol{\Gamma}_{1}\right) \mathbf{A}^{*}\right)+\mathbf{A}^{*} \\
& \stackrel{(b)}{=}\left(\mathbf{I}+\boldsymbol{\Lambda}_{L} \boldsymbol{\Gamma}_{1}\right)^{-1} \boldsymbol{\Lambda}_{L}+\mathbf{A}^{*} \\
& =\left(\boldsymbol{\Lambda}_{L}^{-1}\left(\mathbf{I}+\boldsymbol{\Lambda}_{L} \boldsymbol{\Gamma}_{1}\right)\right)^{-1}+\mathbf{A}^{*} \\
& =\left(\boldsymbol{\Lambda}_{L}^{-1}+\boldsymbol{\Gamma}_{1}\right)^{-1}+\mathbf{A}^{*}
\end{aligned}
$$


where (a) and (b) are from $\Gamma_{1} \mathbf{A}^{*}=\mathbf{0}$.

$$
\begin{aligned}
\frac{\left|\mathbf{D}_{0}^{*}+\mathbf{K}_{z}\right|}{\left|\mathbf{D}_{0}^{*}\right|} & =\left|\mathbf{I}+\mathbf{D}_{0}^{*-1} \mathbf{K}_{z}\right| \\
& =\left|\mathbf{I}+\left(\mathbf{D}_{0}^{-1}+\boldsymbol{\Gamma}_{1}\right) \mathbf{K}_{z}\right| \\
& =\left|\mathbf{I}+\mathbf{D}_{0}^{-1} \mathbf{K}_{z}+\boldsymbol{\Gamma}_{1} \mathbf{K}_{z}\right| \\
& =\left|\mathbf{I}+\mathbf{D}_{0}^{-1} \mathbf{K}_{z}+\boldsymbol{\Gamma}_{1}\left(\left(\mathbf{I}-\mathbf{A}^{*}\right)^{-1}-\mathbf{I}\right)\right| \\
& \stackrel{(c)}{=}\left|\mathbf{I}+\mathbf{D}_{0}^{-1} \mathbf{K}_{z}+\boldsymbol{\Gamma}_{1}\left(\mathbf{I}-\mathbf{A}^{*}\right)\left(\left(\mathbf{I}-\mathbf{A}^{*}\right)^{-1}-\mathbf{I}\right)\right| \\
& =\left|\mathbf{I}+\mathbf{D}_{0}^{-1} \mathbf{K}_{z}\right| \\
& =\frac{\left|\mathbf{D}_{0}+\mathbf{K}_{z}\right|}{\left|\mathbf{D}_{0}\right|},
\end{aligned}
$$

where (c) is from $\Gamma_{1} \mathbf{A}^{*}=\mathbf{0}$.

\section{References}

[1] L. Ozarow, "On a source-coding problem with two channels and three receivers," Bell Syst. Tech. J., vol. 59, no. 10, pp. 1909-1921, Dec. 1980.

[2] A. E. Gamal and T. M. Cover, "Achievable rates for multiple descriptions," IEEE Trans. Inform. Theory, vol. 28, no. 6, pp. 851-857, Nov. 1982.

[3] R. Ahlswede, "The rate-distortion region for multiple descriptions without excess rate," IEEE Trans. Inform. Theory, vol. 31, no. 6, pp. 721-726, Nov. 1985.

[4] T. M. Cover and J. A. Thomas, Elements of Information Theory. New York: Wiley, 1991.

[5] Z. Zhang and T. Berger, "New results in binary multiple descriptions," IEEE Trans. Inform. Theory, vol. 33, no. 4, pp. 502-521, July 1987.

[6] R. Venkataramani, G. Kramer, and V. K. Goyal, "Multiple description coding with many channels," IEEE Trans. Inform. Theory, vol. 49, no. 9, pp. 2106-2114, Sept. 2003.

[7] S. S. Pradhan, R. Puri, and K. Ramchandran, "n-channel symmetric multiple descroption-part I: (n,k) source-channel erasure codes," IEEE Trans. Inform. Theory, vol. 50, no. 1, pp. 47-61, Jan. 2004.

[8] R. Puri, S. S. Pradhan, and K. Ramchandran, "n-channel symmetric multiple descroptionpart II: an achievable rate-distortion region," IEEE Trans. Inform. Theory, vol. 51, no. 4, pp. 1377-1392, Apr. 2005. 
[9] S. Diggavi and T. M. Cover, "Worst additive noise under covariance constraints," IEEE Trans. Inform. Theory, vol. 47, no. 7, pp. 3072-3081, Nov. 2001.

[10] F. Zhang, Matrix Theory: Basic Results and Techniques. Springer, 1999.

[11] H. Wang and P. Viswanath, "Vector Gaussian Multiple Description with Individual and Central Receivers," to appear in IEEE Trans. Inform. Theory, 2007 (available at "http://www.ifp.uiuc.edu/ pramodv/pubs.html").

[12] S Boyd and L. Vandenberghe, Convex Optimization, Cambridge University Press, Cambridge, UK, 2004.

[13] J. Chen and T. Berger. Robust distributed source coding. Submitted to IEEE Trans. Inform. Theory, 2005. 